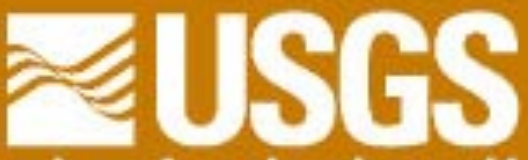

science for a changing world

In cooperation with the Oswego County Department of Health

\title{
Ground-Water Age Dating in Community Wells in Oswego County, New York
}

Open-File Report 01-232

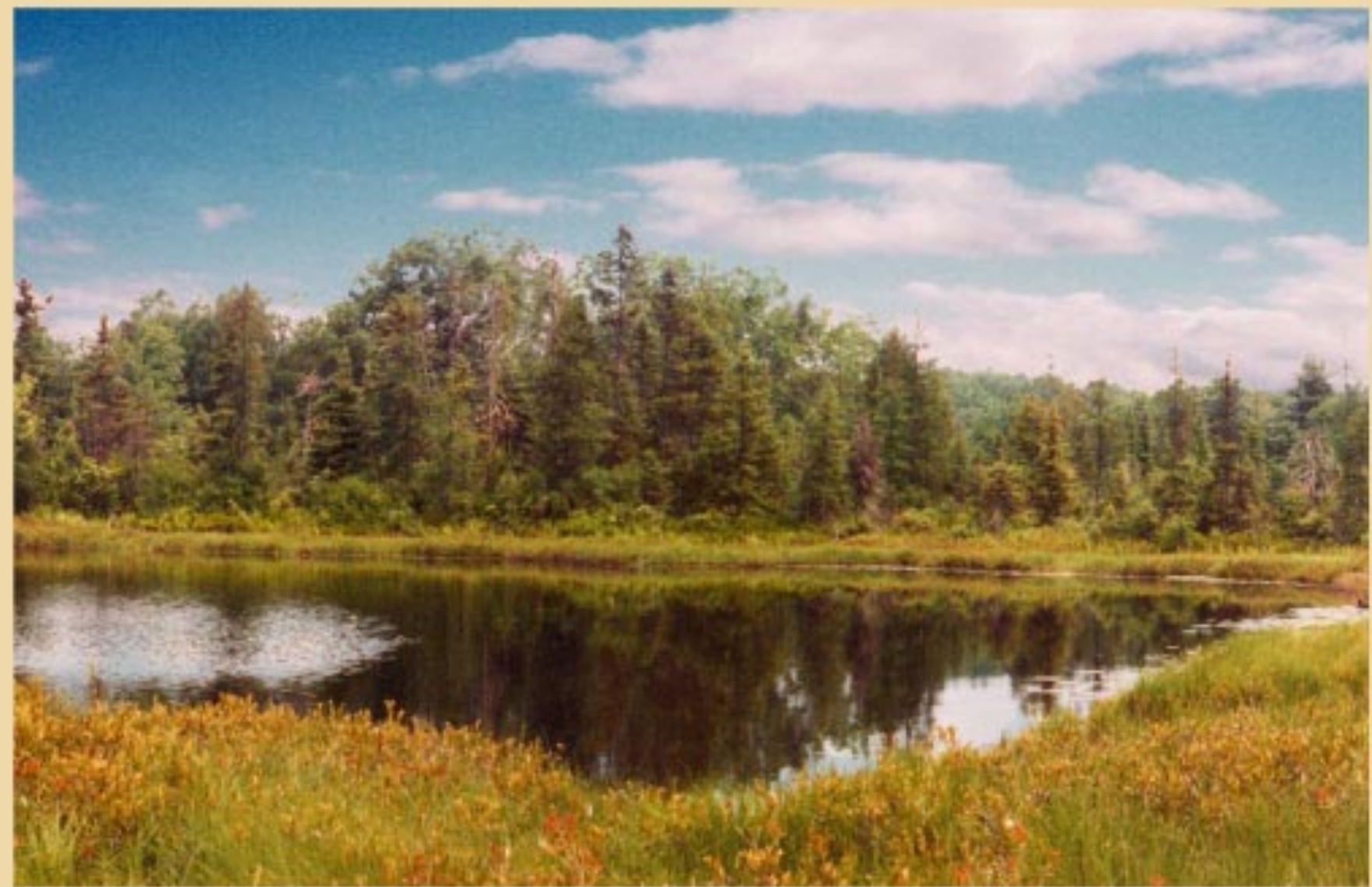

U.S. Department of the Interior

U.S. Geological Survey 
This page has been left blank intentionally. 


\section{Ground-Water Age Dating in Community Wells in Oswego County, New York}

By Stephen C. Komor

U.S. GEOLOGICAL SURVEY

OPEN-FILE REPORT 01-232

In cooperation with the Oswego County

Department of Health

Ithaca, New York

2002 


\section{U.S. DEPARTMENT OF THE INTERIOR \\ Gail A. Norton, Secretary}

U.S. GEOLOGICAL SURVEY

Charles G. Groat, Director

The use of firm, trade, and brand names in this report is for identification purposes

only and does not constitute endorsement by the U.S. Geological Survey.

For additional information write to:

Subdistrict Chief

U.S. Geological Survey

30 Brown Road

Ithaca, NY 14850
Copies of this report can be purchased from:

U.S. Geological Survey

Branch of Information Services

Box 25286

Denver, CO 80225-0286 


\section{CONTENTS}

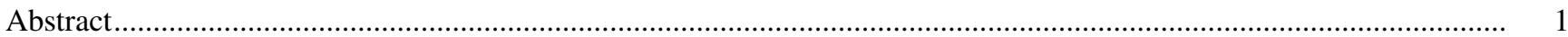

Introduction

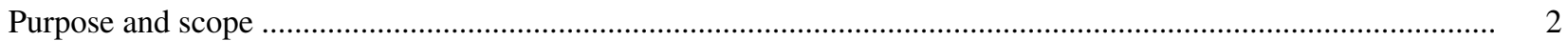

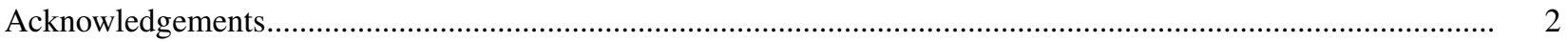

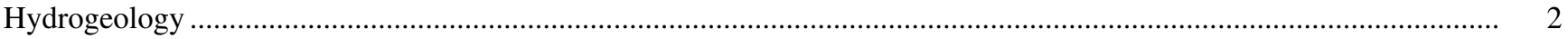

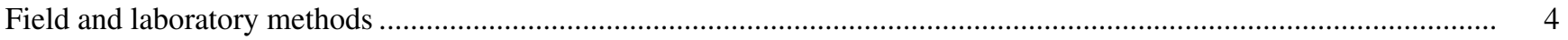

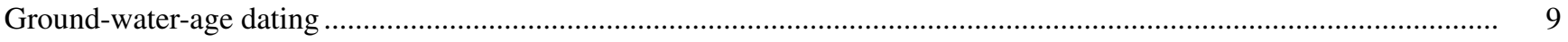

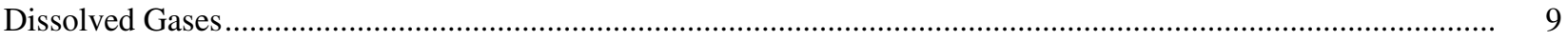

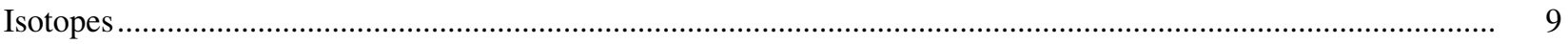

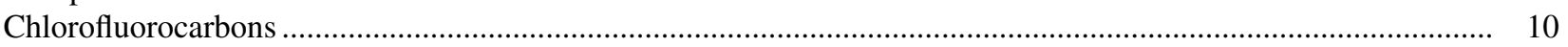

Relations among well depth, ground-water ages, and aquifer lithology .......................................................... 11

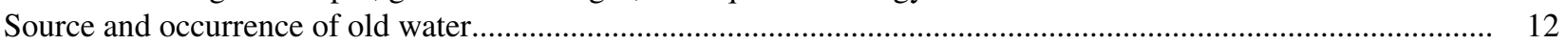

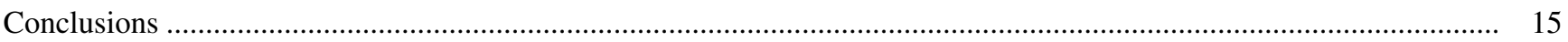

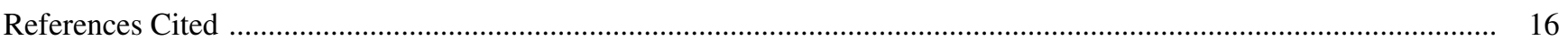

Figures

1. Map showing principal geographic features of Oswego County, N.Y., locations of ground-water sampling sites, and aquifer lithology at well-completion depths of wells sampled in 1999.

2. Concentrations of argon and $\mathrm{N}_{2}$ in ten ground-water samples from community wells in Oswego County

3. Oxygen- and hydrogen-isotope compositions of ground water and precipitation samples collected from selected sites in Oswego County.

4. Comparison of CFC-11 versus CFC-113 concentrations for ground water in Oswego County …............................... 12

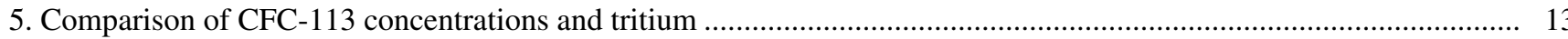

6. Mean ground-water age as a function of well depth and aquifer lithology, at 24 wells in Oswego County .................. 14

7. Conceptual east-west cross-section through Oswego County, N.Y., showing local and subregional flowpaths, and various recharge scenarios

\section{Tables}

1. Data on wells and ground-water samples collected in Oswego County, N.Y. 


\section{CONVERSION FACTORS AND VERTICAL DATUM}

\begin{tabular}{rll}
\hline Multiply & \multicolumn{1}{c}{ By } & To Obtain \\
& Length & \\
centimeter & 0.394 & inch (in.) \\
meter & 3.281 & foot (ft) \\
kilometer & 0.622 & mile (mi) \\
& Area & \\
& 0.386 & \\
square kilometer & & square mile (mi $\left.{ }^{2}\right)$ \\
& & \\
& & Temperature \\
${ }^{\circ} \mathrm{F}=9 / 5{ }^{\circ} \mathrm{C}+32$ & & degrees Fahrenheit \\
& &
\end{tabular}

Specific Conductance

microsiemens per centimeter at $25^{\circ}$ Celsius $(\mu \mathrm{S} / \mathrm{cm})$

Equivalent Concentration Terms

milligrams per liter $(\mathrm{mg} / \mathrm{L})=$ parts per million micrograms per liter $(\mu \mathrm{g} / \mathrm{L})=$ parts per billion

Vertical datum: In this report "sea level" refers to the National Geodetic Vertical Datum of 1929 (NGVD of 1929)—a geodetic datum derived from a general adjustment of the first-order level nets of the United States and Canada, fromerly called Sea Level Datum of 1929. 


\title{
Ground-Water Age Dating in Community Wells in Oswego County, New York
}

\author{
By Stephen C. Komor
}

ABSTRACT

Officials in Oswego County, in north-central New York, have been concerned about potential contamination of community wells. Many of these wells are completed in unconfined glacial sand-and-gravel aquifers, although some are finished in till or in the underlying fractured and jointed bedrock of Late Ordovician and Early Silurian ages. Local shallow ground-water flow is affected by the orientation and hydraulic characteristics of the local topography and surficial sediments, whereas deeper regional flow is toward Lake Ontario. Concentrations of chlorofluorocarbons and tritium in water samples from 28 wells in the county were measured in 1999 for ground-water-age dating; results yield recharge dates ranging from about 1955 to 1994.

The presence of water older than about 15 years in the sand-and-gravel aquifers differs from previous concepts of recharge sources and ground-water movement that were based on numerical modeling of ground-water flow. Young ground water ( 1 to 5 years old) probably

represents recharge from recent precipitation and seepage from streams, whereas the oldest ground water (more than 40 years old) probably is derived from the fractured bedrock that underlies the glacial sediments or has moved along long flow paths in unconsolidated deposits, or through poorly permeable material. Some sand-and-gravel aquifers in Oswego County contain mixtures of old and young water. Wellhead-protection efforts need to focus on protection of the quality of young water in the sand-and-gravel aquifers because young water is more likely to be contaminated than old water.

\section{INTRODUCTION}

Contamination of public water-supply wells has become an increasing concern in the United States in recent years. The Safe Drinking Water Act (U.S.Environmental Protection Agency, 1974) resulted in Federal mandates for protection of public water-supply wells (U.S.Environmental Protection Agency, 1986). The mandate for New York State to institute wellhead-protection strategies for publicsupply wells is enforced mostly by State and county health departments. "Wellhead protection" programs are designed to prevent contamination of drinkingwater supply wells. Such programs delineate the ground-water-contributing areas (recharge zones) of wells, estimate the traveltimes of ground water from its source area to wells, and develop criteria for land management within the recharge zones. Contributing areas can be delineated, and ground-water traveltimes estimated, from finite-difference modeling and particle-tracking simulations with computer programs such as MODFLOW and MODPATH (McDonald and Harbaugh, 1988; Pollock, 1989). The accuracy of results from these numerical methods depends on the accuracy and inherent errors of the input parameters, which include aquifer boundaries, hydraulic characteristics, potentiometric surfaces, and pumping rates. 
The reliability of model results can be evaluated through comparisons with aquifer-recharge dates estimated from chemical characteristics of the water. The most reliable indicators of recent ground-water ages are chlorofluorocarbons (CFCs), $\mathrm{SF}_{6}$, and tritium $\left({ }^{3} \mathrm{H}\right)$ concentrations in the water (Busenberg and Plummer, 1992; Dunkle and others, 1993; Plummer and others, 1993; Reilley and others, 1994; Szabo and others, 1996; Plummer and Busenberg, 2000). CFCs and tritium are man-made substances that have been introduced into the atmosphere-tritium in the late 1950 's and early 1960's by thermonuclear weapons tests; and CFCs since their production in the 1930s, although current production is considered to be less than half of the peak values of the late 1980s.

In 1999, the U.S. Geological Survey (USGS), in cooperation with the Oswego County Department of Health, began a 2-year study to estimate the recharge age of ground water in county aquifers tapped by community wells. The age information can be used to help define ground-water contributing areas. To this end, 28 wells (fig.1.) were sampled in 1999 for CFCs and tritium, and their concentrations were measured to provide a general inventory of ground-water ages at public supply wells in small communities. The sampled wells were at trailer parks, restaurants, small hotels, recreational camps, a golf course, and a power station. Most of these facilities are in unsewered rural settings and could be susceptible to contamination from septic waste. Other potential sources of groundwater contamination within the county include accidental spills of toxic substances; leakage from chemical storage tanks; farm runoff carrying fertilizers, manure, and pesticides; land spreading of sewage sludge; leaking landfills; road salt; and buried pipelines. In general, most contaminants are concentrated near land surface and are carried to the water table by rain and snowmelt that infiltrate the soils. In unconfined aquifers, younger ground water is more likely to contain contaminants in higher concentrations than the deeper, older water, in which contaminants have had more time to be diluted, adsorbed, filtered out, or chemically degraded.

\section{Purpose and Scope}

This report (1) discusses the hydrogeology of Oswego County, (2) summarizes the field and laboratory methods employed, and (3) presents and interprets the results of the ground-water age dating. It also discusses the relations among well depth, groundwater age, and aquifer material, and explains how these features relate to the recharge areas surrounding community supply wells.

The study results are based on samples from 28 community wells in Oswego County. Ground-water ages in each well were estimated from CFC and tritium concentrations. The samples came from a range of aquifer lithologies, including sand-andgravel, sand, silt and clay, and fractured bedrock.

\section{Acknowledgments}

The author thanks Auralie Ashley-Marx, the Oswego County Environmental Program Coordinator, and Chris Williams, Senior Sanitarian, of the Oswego County Health Department; and USGS employees Neil Plummer, Ed Busenberg, Douglas Burns, Jerry Casile, Steven McInnes, and Todd Miller. Thanks also to the many people in Oswego County who granted access to their wells.

\section{HYDROGEOLOGY}

Oswego County encompasses $2,670 \mathrm{~km}^{2}$ on the southeastern shore of Lake Ontario (fig. 1). The climate is humid continental with an average air temperature of about $9^{\circ} \mathrm{C}$. Average annual precipitation (rain plus snow equivalent) ranges from about $97 \mathrm{~cm}$ in the west to $117 \mathrm{~cm}$ in the east (http:// www.worldclimate.com). Surficial unconsolidated glacial deposits throughout the county form hummocky terrains that include: kames and eskers that contain sand and gravel; drumlins that contain lodgment till derived from local bedrock; ablation tills that include a poorly sorted assemblage of silt-to boulder-sized sediments; beach and wave-delta sand and gravel; and proglacial deposits of fine sand, silt, and clay. In places, sand-and-gravel deposits rest directly on bedrock (Miller, 1982). The area contains many local flow systems with distinct hydrologic characteristics and orientations that depend on local topography and sediment type.

Wells completed in clay, silt, and till generally yield 1 to $50 \mathrm{~L} / \mathrm{min}$. Ground-water velocity in these units ranges from about $0.0003 \mathrm{~m} / \mathrm{yr}$ in clayey silt to 9 $\mathrm{m} / \mathrm{yr}$ in fine sand (Miller, 1982). The most productive aquifers in the county are coarse sand-and-gravel deposits that range in thickness from about $3 \mathrm{~m}$ to 38 


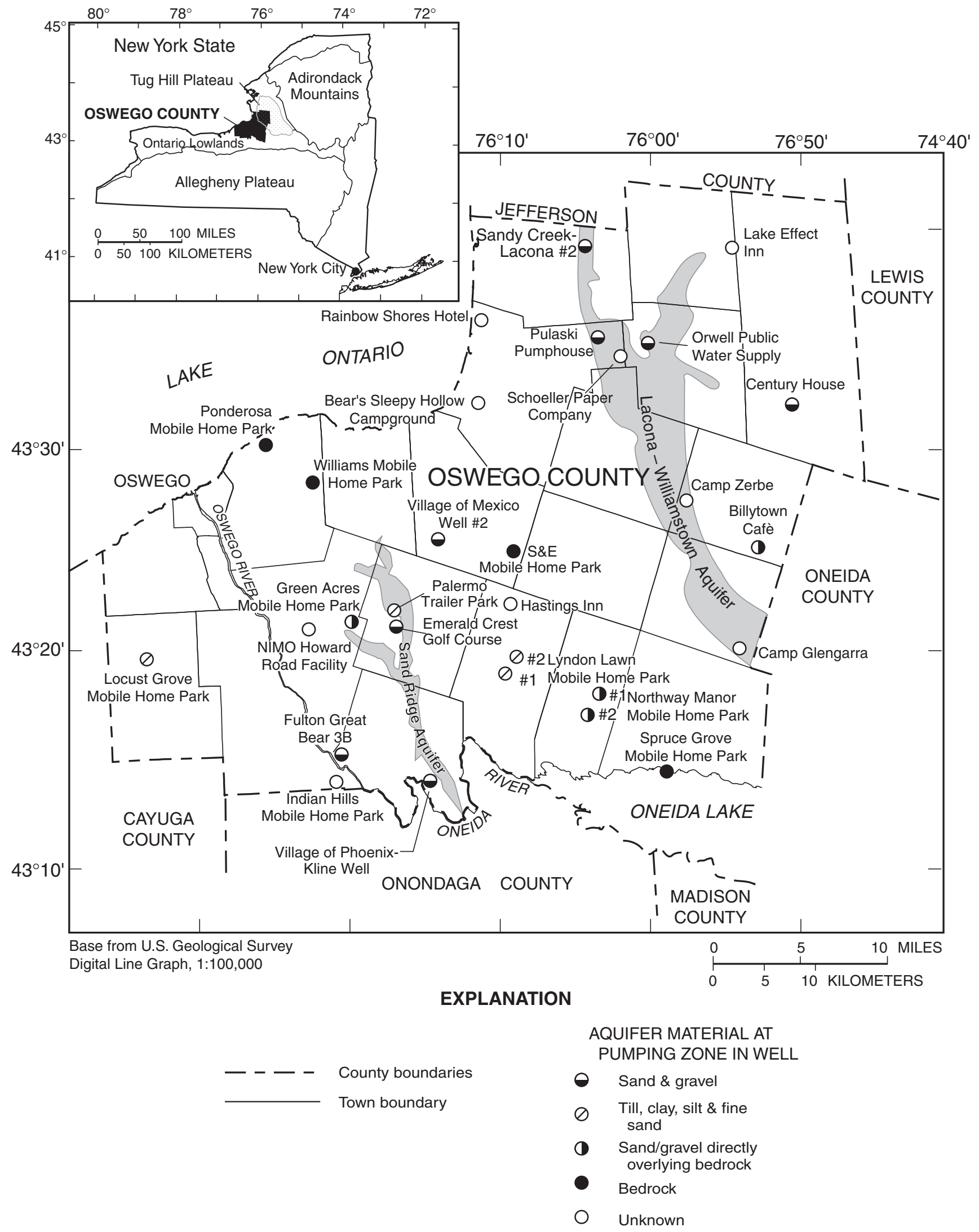

Figure 1. Principal geographic features of Oswego County, N.Y., locations of ground-water sampling sites, and aquifer lithology at well-completion depths of wells sampled in 1999. (Lithology data from drillers' logs and owners' comments.) 
$\mathrm{m}$; wells completed in these deposits yield from about 400 to 3,000 L/min. The two largest sand-and-gravel aquifers are the Lacona-Williamstown aquifer, which is about $32 \mathrm{~km}$ long, 0.5 to $5 \mathrm{~km}$ wide, and 3 to $26 \mathrm{~m}$ thick; and the Sand Ridge aquifer, which is slightly smaller but has similar hydrologic characteristics (Miller and Sherwood, 1993, fig. 1).

The bedrock beneath the glacial deposits is Late Ordovician and Early Silurian shale, siltstone, and sandstone units that dip gently to the southwest. The upper surface of the bedrock, where fractured, forms aquifers. Well yields from bedrock depend on the density, orientation, and width of fractures and joints. Some bedrock aquifers are under artesian pressure; that is, water rises above the bedrock surface (Miller, 1982). Regional ground-water flow in bedrock in the eastern two-thirds of the county generally is southsouthwestward to Lake Ontario (Kantrowitz, 1970; Miller, 1982); that in the southwestern part, west of the Oneida River, is northward to the Lake. Lake Ontario has a mean water level of $74.8 \mathrm{~m}$ above sea level. The Tug Hill Plateau, on the east side of Oswego County (fig. 1, inset map), ranges from $150 \mathrm{~m}$ above sea level at its edges to about $610 \mathrm{~m}$ above sea level at its highest point. Bedrock crops out in some parts of this topographic high or is within about $1 \mathrm{~m}$ of the surface. Precipitation on the plateau is about $150 \mathrm{~cm} / \mathrm{yr}$, much of which is snow that falls primarily between November and January (http://www.worldclimate. com). Snowmelt may be an important source of recharge for the basal bedrock aquifer.

\section{FIELD AND LABORATORY METHODS}

The aquifer lithology at the completion depth of 20 of the 28 sampled wells is given in table 1; the lithology at 8 wells is unknown. The known well depths range from 3.1 to $61.0 \mathrm{~m}$. Many wells are cased to the completion depth; others have screens of variable lengths in the producing units. Some shallow wells are dug cisterns. The S\&E Mobile Home Park has two wells that connect at the wellhead; one well is $23.0 \mathrm{~m}$ deep and completed in fractured bedrock, and the other is $25.9 \mathrm{~m}$ deep and completed in sand and gravel that directly overlies bedrock; thus, samples from this wellhead are a mixture of water from both wells.

Latitude, longitude and elevation of well-sampling locations (fig 1; table 1) were determined by differential global positional satellite (GPS) measurements corrected by LORAN transmissions from a nearby station. The accuracy in all three coordinates is better than $0.1 \mathrm{~m}$.

Values of $\mathrm{pH}$, specific conductance, water temperature, and dissolved-oxygen concentration were monitored with a Hydrolab ${ }^{1} \mathrm{H}_{2} \mathrm{O}$ sensor in a flowthrough cell. The $\mathrm{pH}$, specific conductance, and dissolved-oxygen probes were calibrated daily. Fieldmeasured dissolved-oxygen concentrations averaged $5.5 \pm 2.5 \mathrm{mg} / \mathrm{L}$, whereas laboratory-measured concentrations averaged $4.3 \pm 2.7 \mathrm{mg} / \mathrm{L}$. Field measurements were allowed to stabilize before samples were collected for analysis of CFCs, ${ }^{3} \mathrm{H}$, and nitrate.

Sample water for CFC measurements was pumped through copper tubing attached to hose fittings at the wellheads, upstream from chlorinators, water softeners, pressurized storage tanks, or other equipment. The copper tubing was used to avoid contamination by chlorofluorocarbons that might leach from plastic or Teflon tubing. Samples for chlorofluorocarbon analyses were collected in five glass ampoules that were pre-flushed with ultrapure nitrogen $\left(99.999 \% \mathrm{~N}_{2}\right)$ for 1 min to exclude atmospheric gases. Ampoules were then flushed with sample water for $3 \mathrm{~min}$, before being filled and sealed with a welding torch.

Samples for dissolved $\mathrm{N}_{2}, \mathrm{Ar}, \mathrm{O}_{2}, \mathrm{CO}_{2}$ and $\mathrm{CH}_{4}$ analysis were collected at 12 sites for this study by submerging glass bottles in a plastic bucket that was continuously filled to overflowing with well water. The bottles were filled from the bottom up through the copper tube. Water that was displaced when a stopper was inserted in the bottle escaped through a syringe needle that pierced the stopper. The needle was withdrawn when the bottle was completely full and the stopper firmly in place. The bottles were stored on ice and sent to USGS laboratories in Reston, Va., for analysis.

Concentrations of CFC-12, CFC-11, and CFC-113 were measured in the USGS CFC Laboratory in

Reston, Va. Analyses were done by purge-and-trap gas chromatography and an electron-capture detector. The sensitivity of this equipment allowed measurements at picogram per kilogram ( $\mathrm{pg} / \mathrm{kg}$ ) levels (Busenberg and Plummer, 1992). Analytical uncertainties are approximately $3 \%$ for concentrations in excess of 50

\footnotetext{
${ }^{1}$ Use of trade names is for identification purposes only and does not constitute endorsement by the U.S. Geological Survey.
} 
Table 1. Data on wells and ground-water samples collected in Oswego County, N.Y.

[Dash indicates value is unknown or undetermined; ${ }^{\circ} \mathrm{C}$, degrees Celsius; $\mathrm{CFC}$, chlorofluorocarbon; $\left({ }^{\circ} / \mathrm{oo}\right)$, per mil; $\mu \mathrm{S} / \mathrm{cm}$, microsiemens per centimeter;

$\mathrm{L} / \mathrm{min}$, liters per minute; $\mathrm{mg} / \mathrm{L}$, milligrams per liter: TU, tritium units; pptv, parts per trillion by volume; pg/kg, picograms per kilogram. Well locations are shown in fig. 1$]$

\begin{tabular}{|c|c|c|c|c|c|c|c|c|c|c|c|}
\hline \multirow[b]{2}{*}{ Sampling location } & \multicolumn{2}{|c|}{ Sampling date } & \multicolumn{2}{|c|}{$\begin{array}{c}\text { Location } \\
\text { (decimal degrees) }\end{array}$} & \multirow{2}{*}{$\begin{array}{c}\text { Altitude } \\
\text { (meters } \\
\text { above } \\
\text { sea level) }\end{array}$} & \multirow[b]{2}{*}{$\begin{array}{l}\text { Well depth (meters) and } \\
\text { aquifer composition }\end{array}$} & \multicolumn{5}{|c|}{ Field measurements } \\
\hline & $\begin{array}{l}\text { Tritium } \\
\text { (mo/d/yr) }\end{array}$ & $\begin{array}{l}\text { CFC } \\
(\mathrm{mo} / \mathrm{d} / \mathrm{yr})\end{array}$ & Latitude & Longitude & & & $\begin{array}{l}\text { Water } \\
\text { temp }\left(^{\circ} \mathrm{C}\right)\end{array}$ & $\begin{array}{c}\text { Specific } \\
\text { cond. } \\
(\mu \mathrm{S} / \mathrm{cm})\end{array}$ & $\begin{array}{c}\text { Diss. } \\
\text { oxygen } \\
\text { (mg/L) }\end{array}$ & $\mathrm{pH}$ & $\begin{array}{l}\text { Nitrate } \\
\text { (mg/L) }\end{array}$ \\
\hline Bear's Sleepy Hollow Campground & $8 / 23 / 99$ & $8 / 23 / 99$ & 43.54 & 76.19 & 81.7 & 27.4 & 13.2 & 946 & 1.5 & 7.2 & 2.3 \\
\hline Billytown Cafe $^{1}$ & $8 / 25 / 99$ & $8 / 25 / 99$ & 43.42 & 75.89 & 180.4 & 30.5 ; sand overlying bedrock & 10.9 & 952 & 2.0 & 7.8 & 3.9 \\
\hline Camp Glengarra $^{2}$ & $8 / 26 / 99$ & $8 / 26 / 99$ & 43.34 & 75.90 & 172.7 & 13.7 & 12.5 & 111 & 7.1 & 5.9 & 2.4 \\
\hline Camp Zerbe ${ }^{3}$ & $8 / 24 / 99$ & $8 / 24 / 99$ & 43.46 & 75.96 & 207.7 & 4.6 & 12.7 & 562 & -- & 6.0 & 2.7 \\
\hline Century House ${ }^{3}$ & $8 / 24 / 99$ & $8 / 24 / 99$ & 43.53 & 75.82 & 290.2 & 4.6; sand and gravel & 19.1 & 520 & 2.7 & 6.8 & 4.3 \\
\hline Fulton Great Bear 3B & $2 / 8 / 99$ & $5 / 19 / 99$ & 43.26 & 76.36 & 123.2 & 27.4; sand and gravel & 10.0 & 486 & 3.1 & -- & 1.1 \\
\hline Emerald Crest Golf Course & $8 / 25 / 99$ & $8 / 25 / 99$ & 43.36 & 76.28 & 132.4 & 3.1; sand and gravel & 11.3 & 800 & 8.1 & 7.3 & 5.5 \\
\hline Green Acres Mobile Home Park & $2 / 4 / 99$ & $6 / 3 / 99$ & 43.36 & 76.34 & 150.1 & $\begin{array}{l}\text { 20.7; sand overlying } \\
\text { sandstone bedrock }\end{array}$ & 11.2 & 223 & 5.4 & 8.1 & 2.3 \\
\hline Hastings $\operatorname{Inn}^{3}$ & $8 / 25 / 99$ & $8 / 25 / 99$ & 43.37 & 76.15 & 142.4 & -- & 20.5 & 1198 & 1.7 & 6.4 & 4.4 \\
\hline Indian Hills Mobile Home Park & $2 / 8 / 99$ & $5 / 26 / 99$ & 43.23 & 76.36 & 127.2 & 54.9 & 10.6 & 294 & 0.9 & 7.5 & 2.0 \\
\hline Lake Effect Inn ${ }^{4}$ & $8 / 24 / 99$ & $8 / 24 / 99$ & 43.65 & 75.90 & 417.5 & -- & 12.2 & 343 & 2.0 & 7.6 & 1.2 \\
\hline Locust Grove Mobile Home Park & 8/23/99 & $8 / 23 / 99$ & 43.33 & 76.57 & 121.5 & 5.5 ; silt and clay & 14.2 & 464 & 5.7 & 6.8 & 5.6 \\
\hline Lyndon Lawn Mobile Home Park \#1 & 2/3/99 & $5 / 24 / 99$ & 43.32 & 76.15 & 154.2 & 38.7; clay, sand, and till & 11.1 & 413 & 4.8 & 7.5 & 3.2 \\
\hline Lyndon Lawn Mobile Home Park \#2 & $2 / 3 / 99$ & $5 / 24 / 99$ & 43.32 & 76.15 & 145.7 & 22.6; clay, sand, and till & 10.0 & 319 & 9.1 & 8.0 & 3.2 \\
\hline
\end{tabular}

1. Deep water (short pumping time) has $0.8 \mathrm{TU}$ and no CFCs, which suggest that it recharged before 1955 . Shallow water (long pumping time) contains $522.6 \mathrm{pg} / \mathrm{kg}$ CFC-11 and $215.3 \mathrm{pg} / \mathrm{kg}$ CFC-12, which suggests recharge about 1985. These data imply that young, shallow CFC-contaminated water is drawn down by prolonged pumping. Results from short and long pumping times are shown in figure 6.

2. Probable excess $\mathrm{CFC}-113$; age determined from plot of $\mathrm{CFC}-12$ in relation to ${ }^{3} \mathrm{H}$ (not shown).

3. Probable excess CFC-11; age determined from figure 5.

4. Probable degraded CFC-113; age determined from plot of CFC-12 in relation to ${ }^{3} \mathrm{H}$ (not shown).

5. Two wells - one completed in bedrock; the other in gravel overlying bedrock; the two pump simultaneously into a single wellhead.

6. Contaminated with methane; not used for calculations or plotting on figures 4-6; tritium data suggest recharge in mid-1950's.

7. If information is available.

8. $9^{\circ} \mathrm{C}$ if no $\mathrm{N}_{2} /$ Ar temperature. 
Table 1. Data on wells and ground-water samples collected in Oswego County, N.Y. (continued)

\begin{tabular}{|c|c|c|c|c|c|c|c|c|c|c|c|c|c|c|c|c|c|c|}
\hline \multirow[b]{2}{*}{ Sampling location } & \multicolumn{9}{|c|}{ Laboratory measurements } & \multirow[b]{2}{*}{$\begin{array}{l}\text { CFC } \\
\text { calcu- } \\
\text { lation } \\
\text { temp. } \\
\left({ }^{\circ} \mathrm{C}\right) \\
\end{array}$} & \multicolumn{2}{|c|}{ Isotope value } & \multicolumn{3}{|c|}{$\begin{array}{l}\text { Calculated CFC } \\
\text { concentrations in air }\end{array}$} & \multirow[b]{2}{*}{$\begin{array}{l}\text { Tritium } \\
\text { con- } \\
\text { cen- } \\
\text { tration } \\
\text { (TU) }\end{array}$} & \multicolumn{2}{|c|}{ Model recharge dates } \\
\hline & $\begin{array}{c}\mathrm{N}_{2} \\
(\mathrm{mg} / \mathrm{L}) \\
\end{array}$ & $\begin{array}{c}\mathrm{Ar} \\
(\mathrm{mg} / \mathrm{L}) \\
\end{array}$ & $\begin{array}{c}\mathrm{O}_{2} \\
(\mathrm{mg} / \mathrm{L}) \\
\end{array}$ & $\begin{array}{l}\mathrm{CO}_{2} \\
(\mathrm{mg} / \mathrm{L}) \\
\end{array}$ & $\begin{array}{l}\mathrm{CH}_{4} \\
(\mathrm{mg} / \mathrm{L}) \\
\end{array}$ & $\begin{array}{l} \\
\mathrm{N}_{2} / \mathrm{Ar} \\
\text { temp. } \\
\left.{ }^{\circ} \mathrm{C}\right)\end{array}$ & $\begin{array}{c}\text { CFC-11 } \\
\text { (pg/kg; } \\
\text { mean.- } \\
\text { std. } \\
\text { dev.) }\end{array}$ & $\begin{array}{l}\text { CFC -12 } \\
\text { (pg/kg; } \\
\text { mean.- } \\
\text { std. } \\
\text { dev.) }\end{array}$ & $\begin{array}{l}\text { CFC-113 } \\
\text { (pg/kg; } \\
\text { mean.- } \\
\text { std. } \\
\text { dev.) } \\
\end{array}$ & & $\begin{array}{l}\delta^{18} \mathrm{O} \\
(\%)\end{array}$ & $\begin{array}{l}\delta D \\
(\%) \\
\end{array}$ & $\begin{array}{c}\text { CFC-11 } \\
\text { (pptv) }\end{array}$ & $\begin{array}{c}\text { CFC - } \\
12 \\
(p p t v) \\
\end{array}$ & $\begin{array}{l}\text { CFC- } \\
113 \\
\text { (pptv) }\end{array}$ & & $\begin{array}{l}\text { Year of recharge } \\
\text { and comments }\end{array}$ & $\begin{array}{c}\text { Median } \\
\text { ground- } \\
\text { water } \\
\text { age } \\
\text { (years) } \\
\end{array}$ \\
\hline $\begin{array}{l}\text { Bear's Sleepy Hollow } \\
\text { Camp-ground }\end{array}$ & 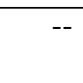 & $\begin{array}{l}-- \\
\end{array}$ & $\begin{array}{l}-- \\
\end{array}$ & $\begin{array}{ll}-- \\
\end{array}$ & $\begin{array}{l}- \\
\end{array}$ & $\begin{array}{l}-- \\
\end{array}$ & $\begin{array}{r}28.7 \\
-2.0\end{array}$ & $\begin{array}{r}1.9 \\
-0.8\end{array}$ & $\begin{array}{r}2.3 \\
-4.0\end{array}$ & 9.0 & $\begin{array}{l}-10.0 \\
\end{array}$ & -66.9 & 9.5 & 2.7 & 1.8 & 15.9 & $1957 \pm 1$ year & 42 \\
\hline Billytown Cafe ${ }^{1}$ & -- & -- & -- & -- & -- & -- & $\begin{array}{r}0 \text { to } \\
522.6\end{array}$ & $\begin{array}{r}0 \text { to } \\
215.3\end{array}$ & $\begin{array}{c}\text { not } \\
\text { detected }\end{array}$ & 9.0 & -11.9 & -91.4 & 215.3 & 319.5 & 0.0 & 0.8 & see note 1 & $14 / 44$ \\
\hline Camp Glengarra $^{2}$ & -- & -- & -- & -- & & -- & $\begin{array}{r}701.6 \\
-25.3\end{array}$ & $\begin{array}{r}445.7 \\
-27.6\end{array}$ & $\begin{array}{r}123.3 \\
-59.0\end{array}$ & 9.0 & -11.7 & -86.0 & 236.2 & 660.8 & 98.0 & 22.5 & $\begin{array}{l}1992 \pm 5 \text { years; excess } \\
\text { CFC }-12 \text { and }-113\end{array}$ & 8 \\
\hline Camp Zerbe ${ }^{3}$ & 18.8 & 0.7 & 4.8 & 53.3 & 0.0 & 9.2 & $\begin{array}{l}16800.6 \\
-632.6\end{array}$ & $\begin{array}{r}412.3 \\
-22.5\end{array}$ & $\begin{array}{r}92.7 \\
-9.7\end{array}$ & 9.2 & -12.5 & -84.5 & 5681.1 & 613.8 & 74.0 & 24.2 & $\begin{array}{l}1989 \pm 3 \text { years; excess } \\
\text { CFC- } 11 \text { and }-12 \text {; elevated } \\
\mathrm{CO}_{2}\end{array}$ & 10 \\
\hline Century House $^{3}$ & -- & -- & -- & -- & -- & -- & $\begin{array}{r}5801.4 \\
-1317.6\end{array}$ & $\begin{array}{r}1094.1 \\
-78.3\end{array}$ & $\begin{array}{r}56.5 \\
-6.2\end{array}$ & 9.0 & -11.9 & -78.3 & 1981.6 & 1645.3 & 45.6 & 26.5 & $\begin{array}{l}1986 \pm 3 \text { years; excess } \\
\text { CFC-11 and }-12 ; \text { possible } \\
\text { mixture }\end{array}$ & 13 \\
\hline Fulton Great Bear 3B & 24.0 & 0.8 & 1.6 & 5.8 & 0.0 & 4.8 & $\begin{array}{r}833.3 \\
-385.1\end{array}$ & $\begin{array}{r}4450.5 \\
-44.1\end{array}$ & $\begin{array}{r}69.0 \\
-3.0\end{array}$ & 4.8 & -- & -- & 21.1 & 178.5 & 0.0 & 25.7 & $1956-62$ & 40 \\
\hline $\begin{array}{l}\text { Emerald Crest Golf } \\
\text { Course }\end{array}$ & -- & -- & -- & -- & -- & -- & $\begin{array}{r}80.6 \\
-0.4\end{array}$ & $\begin{array}{l}151.2 \\
-3.3\end{array}$ & $\begin{array}{r}0.0 \\
-0.0\end{array}$ & 9.0 & -11.9 & -80.7 & 185.0 & 6565.3 & 54.6 & 24.7 & $\begin{array}{l}1982-87 ; \text { excess CFC-12; } \\
\text { probable mixture }\end{array}$ & 14 \\
\hline $\begin{array}{l}\text { Green Acres Mobile } \\
\text { Home Park }\end{array}$ & -- & -- & -- & -- & -- & -- & $\begin{array}{l}122.8 \\
-8.4\end{array}$ & $\begin{array}{l}206.8 \\
-2.0\end{array}$ & $\begin{array}{r}2.6 \\
-0.2\end{array}$ & 9.0 & -- & -- & 38.9 & 290.0 & 1.9 & 26.8 & 1957 - 67; excess CFC-12 & 37 \\
\hline Hastings $\operatorname{Inn}^{3}$ & -- & -- & -- & -- & -- & -- & $\begin{array}{r}1261.9 \\
-13.2\end{array}$ & $\begin{array}{r}1345.7 \\
-18.4\end{array}$ & $\begin{array}{r}110.2 \\
-5.8\end{array}$ & 9.0 & -12.6 & -80.7 & 423.3 & 1987.6 & 87.3 & 22.4 & $\begin{array}{l}1994 \pm 3 \text { years; excess } \\
\text { CFC- } 11 \text { and }-12 ; \\
\mathrm{N}_{2} \mathrm{O} \text { present }\end{array}$ & 5 \\
\hline $\begin{array}{l}\text { Indian Hills Mobile } \\
\text { Home Park }\end{array}$ & -- & -- & -- & -- & -- & -- & $\begin{array}{r}64.1 \\
-0.6\end{array}$ & $\begin{array}{l}119.4 \\
-4.6\end{array}$ & $\begin{array}{r}0.6 \\
-1.1\end{array}$ & 9.0 & -- & -- & 20.3 & 167.5 & 0.5 & 23.7 & $1956-62$ & 40 \\
\hline Lake Effect Inn ${ }^{4}$ & 23.2 & 0.8 & 0.1 & 2.5 & 0.0 & 5.1 & $\begin{array}{r}168.8 \\
-14.1\end{array}$ & $\begin{array}{r}47.3 \\
-0.7\end{array}$ & $\begin{array}{r}0.0 \\
-0.0\end{array}$ & 5.1 & -11.4 & -70.1 & 46.5 & 58.8 & 0.0 & 26.4 & $\begin{array}{l}1970 \pm 3 \text { years; probable } \\
\text { degraded CFC }-113\end{array}$ & 29 \\
\hline $\begin{array}{l}\text { Locust Grove Mobile } \\
\text { Home Park }\end{array}$ & 19.0 & 0.7 & 2.3 & 29.9 & 0.0 & 9.3 & $\begin{array}{r}736.2 \\
-30.3\end{array}$ & $\begin{array}{r}419.4 \\
-10.1\end{array}$ & $\begin{array}{r}72.8 \\
-3.6\end{array}$ & 9.3 & -12.0 & -78.5 & 253.5 & 617.9 & 57.5 & 26.9 & $1987-92$ & 9 \\
\hline $\begin{array}{l}\text { Lyndon Lawn Mobile } \\
\text { Home Park \#2 }\end{array}$ & 22.0 & 0.8 & 6.6 & 1.5 & 0.0 & 7.2 & $\begin{array}{r}517.1 \\
-9.0\end{array}$ & $\begin{array}{r}357.0 \\
-2.8\end{array}$ & $\begin{array}{r}37.2 \\
-3.3\end{array}$ & 7.2 & -- & -- & 156.4 & 480.7 & 26.3 & 24.8 & $\begin{array}{l}1978-82 ; \text { probable } \\
\text { mixture }\end{array}$ & 19 \\
\hline
\end{tabular}


Table 1. Data on wells and ground-water samples collected in Oswego County, N.Y. (continued)

\begin{tabular}{|c|c|c|c|c|c|c|c|c|c|c|c|}
\hline \multirow[b]{2}{*}{ Sampling location } & \multicolumn{2}{|c|}{ Sampling date } & \multicolumn{2}{|c|}{$\begin{array}{c}\text { Location } \\
\text { (decimal degrees) }\end{array}$} & \multirow{2}{*}{$\begin{array}{l}\text { Altitude } \\
\text { (meters } \\
\text { above } \\
\text { sea level) }\end{array}$} & \multirow[b]{2}{*}{$\begin{array}{l}\text { Well depth (meters) and } \\
\text { aquifer composition }\end{array}$} & \multicolumn{5}{|c|}{ Field measurements } \\
\hline & $\begin{array}{l}\text { Tritium } \\
\text { (mo/d/yr) }\end{array}$ & $\begin{array}{c}\mathrm{CFC} \\
(\mathrm{mo} / \mathrm{d} / \mathrm{yr})\end{array}$ & Latitude & Longitude & & & $\begin{array}{l}\text { Water } \\
\text { temp } \\
\left({ }^{\circ} \mathrm{C}\right)\end{array}$ & $\begin{array}{c}\text { Specific } \\
\text { cond. } \\
(\mu \mathrm{S} / \mathrm{cm})\end{array}$ & $\begin{array}{c}\text { Diss. } \\
\text { oxygen } \\
\text { (mg/L) }\end{array}$ & $\mathrm{pH}$ & $\begin{array}{l}\text { Nitrate } \\
(\mathrm{mg} / \mathrm{L})\end{array}$ \\
\hline NIMO Howard Road Facility & $2 / 2 / 99$ & $6 / 3 / 99$ & 43.36 & 76.38 & 141.7 & 54.8 & 12.6 & 605 & 3.8 & 7.1 & 1.1 \\
\hline Northway Manor Mobile Home Park 1 & 2/3/99 & $5 / 25 / 99$ & 43.30 & 76.05 & 161.0 & 61.0 ; sand overlying bedrock & 10.6 & 422 & 2.2 & 7.3 & 1.7 \\
\hline Northway Manor Mobile Home Park 2 & 2/3/99 & $5 / 25 / 99$ & 43.30 & 76.06 & 160.0 & 61.0 ; sand overlying bedrock & 11.2 & 679 & 3.1 & 7.1 & 0.5 \\
\hline Orwell Public Water Supply & $8 / 26 / 99$ & $8 / 26 / 99$ & 43.58 & 75.99 & 268.1 & 6.1 ; sand and gravel & 14.3 & 246 & 7.6 & 6.3 & 6.6 \\
\hline Palermo Trailer Park & 2/8/99 & $5 / 26 / 99$ & 43.37 & 76.28 & 131.1 & 5.5; till & 11.0 & 792 & 0.8 & 8.4 & 3.7 \\
\hline Ponderosa Mobile Home Park & $8 / 23 / 99$ & $8 / 23 / 99$ & 43.50 & 76.43 & 83.3 & 12.2; unspecified bedrock & 12.6 & 1212 & 1.6 & 7.1 & 2.6 \\
\hline Pulaski Pumphouse & $2 / 5 / 99$ & $5 / 27 / 99$ & 43.57 & 76.05 & 151.7 & 4.6; sand and gravel & 8.7 & 228 & 7.7 & 7.7 & 4.5 \\
\hline Rainbow Shores Hotel & $8 / 26 / 99$ & $8 / 26 / 99$ & 43.60 & 76.20 & 78.7 & -- & 11.7 & 355 & 6.7 & 8.7 & 2.8 \\
\hline S\&E Mobile Home Park ${ }^{5}$ & 2/3/99 & $5 / 26 / 99$ & 43.42 & 76.16 & 138.0 & $\begin{array}{l}\text { Two wells pumping simultaneously: } \\
\text { (1) } 23 \text { m deep completed in bedrock; } \\
\text { (2) } 25.9 \text { m deep, completed in } \\
\text { coarse gravel overlying bedrock }\end{array}$ & 9.9 & 366 & 2.4 & 7.4 & 2.9 \\
\hline Sandy Creek Lacona \#2 & $8 / 24 / 99$ & $8 / 24 / 99$ & 43.65 & 76.07 & 164.6 & 6.4; sand and gravel & 18.5 & 285 & 5.4 & 7.1 & 2.6 \\
\hline Schoeller Well B7 & -- & -- & -- & -- & -- & 18.3 & 12.2 & 442 & 1.0 & 7.4 & -- \\
\hline Spruce Grove Mobile Home Park ${ }^{6}$ & 2/3/99 & $5 / 27 / 99$ & 43.24 & 75.98 & 118.7 & 25.9; unspecified bedrock & 10.6 & 393 & 0.8 & 7.4 & 3.1 \\
\hline Village of Mexico Well \#2 & $12 / 29 / 98$ & $5 / 19 / 99$ & 43.43 & 76.23 & 125.0 & 10.7; sand and gravel & 9.9 & 236 & 8.3 & -- & 2.4 \\
\hline Village of Phoenix - Kline well & $12 / 29 / 98$ & $6 / 3 / 99$ & 43.25 & 76.24 & 93.9 & 13.4; sand and gravel & 10.1 & 458 & 1.4 & 7.0 & 2.5 \\
\hline Williams Mobile Home Park ${ }^{6}$ & $8 / 23 / 99$ & $8 / 23 / 99$ & 43.47 & 76.38 & 136.1 & 39.6; unspecified bedrock & 16.0 & 954 & 1.5 & 8.0 & 1.2 \\
\hline
\end{tabular}


Table 1. Data on wells and ground-water samples collected in Oswego County, N.Y. (continued)

\begin{tabular}{|c|c|c|c|c|c|c|c|c|c|c|c|c|c|c|c|c|c|c|}
\hline \multirow[b]{2}{*}{ Sampling location } & \multicolumn{9}{|c|}{ Laboratory measurements } & \multirow[b]{2}{*}{$\begin{array}{l}\text { CFC } \\
\text { calcu- } \\
\text { lation } \\
\text { temp. } \\
\left({ }^{\circ} \mathrm{C}\right)\end{array}$} & \multicolumn{2}{|c|}{ Isotope value } & \multicolumn{3}{|c|}{$\begin{array}{l}\text { Calculated CFC } \\
\text { concentrations in air }\end{array}$} & \multirow[b]{2}{*}{$\begin{array}{l}\text { Tritium } \\
\text { con- } \\
\text { cen- } \\
\text { tration } \\
\text { (TU) }\end{array}$} & \multicolumn{2}{|c|}{ Model results } \\
\hline & $\begin{array}{c}\mathrm{N}_{2} \\
(\mathrm{mg} / \mathrm{L}) \\
\end{array}$ & $\begin{array}{c}\mathrm{Ar} \\
(\mathrm{mg} / \mathrm{L}) \\
\end{array}$ & $\begin{array}{c}\mathrm{O}_{2} \\
(\mathrm{mg} / \mathrm{L}) \\
\end{array}$ & $\begin{array}{r}\mathrm{CO}_{2} \\
(\mathrm{mg} / \mathrm{L}) \\
\end{array}$ & $\begin{array}{c}\mathrm{CH}_{4} \\
(\mathrm{mg} / \mathrm{L}) \\
\end{array}$ & $\begin{array}{l}\mathrm{N}_{2} / \mathrm{Ar} \\
\text { temp } \\
\left({ }^{\circ} \mathrm{C}\right) \\
\end{array}$ & $\begin{array}{l}\text { CFC-11 } \\
\text { (pg/kg; } \\
\text { mean - } \\
\text { std. } \\
\text { dev.) }\end{array}$ & $\begin{array}{c}\text { CFC-12 } \\
\text { (pg/kg; } \\
\text { mean - } \\
\text { std. } \\
\text { dev.) }\end{array}$ & $\begin{array}{c}\text { CFC-113 } \\
\text { (pg/kg; } \\
\text { mean - } \\
\text { std. } \\
\text { dev.) } \\
\end{array}$ & & $\begin{array}{l}\delta^{18} \mathrm{O} \\
(\%) \\
\end{array}$ & $\begin{array}{c}\delta D \\
(\%) \\
\end{array}$ & $\begin{array}{c}\text { CFC-11 } \\
\text { (pptv) }\end{array}$ & $\begin{array}{c}\text { CFC-12 } \\
\text { (pptv) }\end{array}$ & $\begin{array}{c}\text { CFC- } \\
113 \\
\text { (pptv) } \\
\end{array}$ & & $\begin{array}{l}\text { Year of recharge } \\
\text { and comments }\end{array}$ & $\begin{array}{c}\text { Median } \\
\text { ground- } \\
\text { water } \\
\text { age } \\
\text { (years) } \\
\end{array}$ \\
\hline $\begin{array}{l}\text { NIMO Howard Road } \\
\text { Facility }\end{array}$ & -- & --- & --- & -- & 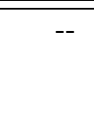 & & $\begin{array}{r}89.9 \\
-29.1\end{array}$ & $\begin{array}{r}548.1 \\
-17.5\end{array}$ & $\begin{array}{r}56.9 \\
-65.2 \\
\end{array}$ & 9.0 & --- & $\begin{array}{l}- \\
\end{array}$ & 28.5 & 768.6 & 14.4 & 29.1 & $\begin{array}{l}1967-76 \text {; excess } \\
\text { CFC-12; } \\
\text { possible mixture }\end{array}$ & 27 \\
\hline $\begin{array}{l}\text { Northway Manor Mobile } \\
\text { Home Park }\end{array}$ & -- & -- & -- & -- & -- & & $\begin{array}{r}81.0 \\
-4.8\end{array}$ & $\begin{array}{l}122.8 \\
-6.6\end{array}$ & $\begin{array}{r}6.8 \\
-0.5\end{array}$ & 9.0 & -- & -- & 25.6 & 172.2 & 5.0 & 23.6 & $1965-72$ & 30 \\
\hline $\begin{array}{l}\text { Northway Manor Mobile } \\
\text { Home Park }\end{array}$ & -- & -- & -- & -- & -- & & $\begin{array}{r}149.3 \\
-42.2\end{array}$ & $\begin{array}{l}180.5 \\
-9.1\end{array}$ & $\begin{array}{r}16.2 \\
-0.1\end{array}$ & 9.0 & -- & -- & 53.9 & 253.1 & 12.0 & 24.0 & $\begin{array}{l}1970-76 \\
\text { possible mixture }\end{array}$ & 26 \\
\hline $\begin{array}{l}\text { Orwell Public Water } \\
\text { Supply }\end{array}$ & 18.3 & 0.7 & 6.0 & 28.3 & 0.0 & 9.6 & $\begin{array}{r}437.8 \\
-389.0\end{array}$ & $\begin{array}{l}363.8 \\
-5.3\end{array}$ & $\begin{array}{r}92.3 \\
-4.8\end{array}$ & 9.6 & -12.5 & -83.7 & 245.7 & 545.6 & 74.2 & 25.0 & $\begin{array}{l}1988-93 \\
\text { possible mixture }\end{array}$ & 8 \\
\hline Palermo Trailer Park & -- & -- & -- & -- & -- & -- & $\begin{array}{r}16.8 \\
-3.5\end{array}$ & $\begin{array}{r}9.3 \\
-1.8\end{array}$ & $\begin{array}{r}6.7 \\
-4.6\end{array}$ & 9.0 & -- & -- & 5.3 & 13.0 & 5.0 & 0.9 & $1955 \pm 5$ years & 44 \\
\hline $\begin{array}{l}\text { Ponderosa Mobile Home } \\
\text { Park }\end{array}$ & -- & -- & -- & -- & -- & -- & $\begin{array}{r}93.6 \\
-111.8\end{array}$ & $\begin{array}{r}38.0 \\
-0.6\end{array}$ & $\begin{array}{r}5.3 \\
-1.7\end{array}$ & 9.0 & -10.3 & -68.8 & 4.0 & 55.7 & 4.1 & 21.3 & $1957 \pm 3$ years & 42 \\
\hline Pulaski Pumphouse & 21.8 & 0.8 & 8.1 & 1.8 & 0.0 & 4.7 & $\begin{array}{r}435.7 \\
-1.7\end{array}$ & $\begin{array}{l}458.2 \\
-6.8\end{array}$ & $\begin{array}{r}18.4 \\
-7.0\end{array}$ & 4.7 & -12.4 & -81.8 & 113.5 & 539.9 & 11.1 & 26.6 & $1975 \pm 2$ years & 24 \\
\hline Rainbow Shores Hotel & -- & -- & -- & -- & -- & -- & $\begin{array}{r}191.1 \\
-43.5\end{array}$ & $\begin{array}{r}176.4 \\
-40.8\end{array}$ & $\begin{array}{r}42.1 \\
-12.3 \\
\end{array}$ & 9.0 & -11.3 & -76.6 & 63.6 & 258.6 & 33.1 & 9.1 & $\begin{array}{l}1972-83 ; \\
\text { possible mixture } \\
\text { with CFC-11 } \\
\text { degradation }\end{array}$ & 21 \\
\hline S\&E Mobile Home Park & -- & -- & -- & -- & -- & -- & $\begin{array}{r}254.9 \\
-0.6\end{array}$ & $\begin{array}{r}749.4 \\
-3.2\end{array}$ & $\begin{array}{r}14.0 \\
-1.0\end{array}$ & 9.0 & -- & -- & 139.1 & 843.3 & 22.3 & 14.3 & $\begin{array}{l}1978 \pm 3 \text { years; } \\
\text { excess CFC- } 12 \\
\text { possible mixture }\end{array}$ & 21 \\
\hline Sandy Creek Lacona \#2 & 21.2 & 0.8 & 4.1 & 6.5 & 0.0 & 8.5 & $\begin{array}{r}299.0 \\
-253.1\end{array}$ & $\begin{array}{l}441.9 \\
-6.2\end{array}$ & $\begin{array}{r}38.3 \\
-3.2\end{array}$ & 8.5 & -11.7 & -77.1 & 162.6 & 654.5 & 30.4 & 25.7 & $\begin{array}{l}1982 \pm 2 \text { years; } \\
\text { excess CFC-12; } \\
\text { probable mixture }\end{array}$ & 17 \\
\hline Schoeller Well B7 & -- & -- & -- & -- & -- & -- & -- & -- & -- & -- & -11.6 & -73.9 & -- & -- & -- & -- & $\begin{array}{l}\text { No CFC or } \\
\text { tritium data }\end{array}$ & -- \\
\hline $\begin{array}{l}\text { Spruce Grove Mobile } \\
\text { Home Park }\end{array}$ & 28.7 & 1.0 & -- & 7.1 & 19.8 & -- & $\begin{array}{r}1.0 \\
-0.3\end{array}$ & $\begin{array}{r}115.1 \\
-22.5\end{array}$ & $\begin{array}{r}0.0 \\
-0.0\end{array}$ & 9.0 & -- & -- & 0.3 & 161.0 & 0.0 & 4.9 & Mid-1950's & 44 \\
\hline $\begin{array}{l}\text { Village of Mexico } \\
\text { Well \#2 }\end{array}$ & 20.4 & 0.8 & 7.2 & 1.3 & 0.0 & 7.1 & $\begin{array}{l}560.3 \\
-4.3\end{array}$ & $\begin{array}{r}350.3 \\
-3.4\end{array}$ & $\begin{array}{r}12.2 \\
-1.3\end{array}$ & 7.1 & -- & -- & 139.5 & 463.1 & 6.0 & 30.5 & $1968-78$ & 22 \\
\hline $\begin{array}{l}\text { Village of Phoenix - } \\
\text { Kline well }\end{array}$ & -- & -- & -- & -- & -- & -- & $\begin{array}{l}129.2 \\
-1.0\end{array}$ & $\begin{array}{r}734.2 \\
-14.9\end{array}$ & $\begin{array}{r}18.4 \\
-0.8\end{array}$ & 9.0 & -10.7 & -75.4 & 33.9 & 873.5 & 11.2 & 28.3 & $\begin{array}{l}1967-72 \text {; excess } \\
\text { CFC-12; } \\
\text { possible mixture }\end{array}$ & 29 \\
\hline $\begin{array}{l}\text { Williams Mobile Home } \\
\text { Park }^{6}\end{array}$ & 13.4 & 0.5 & 0.1 & 2.7 & 20.7 & -- & $\begin{array}{r}19.7 \\
-4.7\end{array}$ & $\begin{array}{r}60.8 \\
-8.8\end{array}$ & $\begin{array}{r}11.6 \\
-3.2\end{array}$ & 9.0 & -12.2 & -79.4 & 6.6 & 89.7 & 9.2 & 5.5 & Mid-1950s & 44 \\
\hline
\end{tabular}


$\mathrm{pg} / \mathrm{kg}$ and approach $50 \%$ at the detection limit of $1 \mathrm{pg} /$ $\mathrm{kg}$. Concentrations of $\mathrm{N}_{2}, \mathrm{Ar}, \mathrm{O}_{2}, \mathrm{CO}_{2}$ and $\mathrm{CH}_{4}$ were measured by gas chromatography.

Twenty-eight water samples for ${ }^{3} \mathrm{H}$ measurements were collected by submerging 1-liter baked, amber glass jars in a plastic bucket that was continuously refilled with fresh well water. Samples were stored on ice and sent to the University of Waterloo

Environmental Isotope Laboratory for analysis by liquid scintillation counting. The detection limit is 0.8 $\mathrm{TU}$, and the analytical error is $1.5 \mathrm{TU}$. Ground water for stable-isotope analyses was collected at 17 sites. Mixed rain and snow samples for stable-isotope analyses were collected near the Schoeller Paper Company well, Pulaski Pump house well, and Village of Phoenix well (fig. 1). Precipitation was sampled at each location was sampled on January 4, 10, and 11, 2000. Average climatic variables during the sampling period were: maximum air temperature, $6.8^{\circ} \mathrm{C}$; minimum air temperature, $-0.4^{\circ} \mathrm{C}$; rainfall, $0.6 \mathrm{~cm}$; and snowfall, $0.2 \mathrm{~cm}$. Precipitation was funneled into a glass jar that contained silicone oil to prevent evaporation. The sampling device was deployed for 24 to 76 hours to integrate storm precipitation.

Stable-isotope measurements were done in the University of Waterloo Environmental Isotope Laboratory. The precision of $\delta^{18} \mathrm{O}$ analyses is $0.05 \%$, and of $\delta \mathrm{D}$ analyses is $0.5 \%$. Ground-water nitrate $\left(\mathrm{NO}_{3}\right)$ concentrations were analyzed in the field with a Hach 2010 portable spectrophotometer. The range of detectable concentrations was 0 to $4.5 \mathrm{mg} / \mathrm{L}$. Twofold dilutions were necessary for three samples whose concentrations exceeded $4.5 \mathrm{mg} / \mathrm{L}$. The analytical precision was $1.0 \pm 0.8 \mathrm{mg} / \mathrm{L}$, whereas blanks had concentrations within $0.1 \mathrm{mg} / \mathrm{L}$ of 0 .

\section{GROUND-WATER-AGE DATING}

Ground water was dated using CFC and tritium $\left({ }^{3} \mathrm{H}\right)$ concentrations. The ages are the approximate time since the water entered the saturated zone during recharge and are based on matching the concentrations of the environmental tracers in ground water to the historical atmospheric concentrations of the tracers. Details of the CFC dating are explained in Busenburg and Plummer (1992), Plummer and others (1993), and Reilly and others (1994). CFC ages assume the CFC composition of the infiltrating ground water is in equilibrium with that of the atmosphere at the time of recharge; the historical atmospheric concentrations of are well documented.
Equilibrium partitioning of CFCs between air and water is temperature and pressure dependent. Dissolved nitrogen and argon from the atmosphere can be used to calculate the temperature at the time of recharge. Tritium dates are estimated by comparing ${ }^{3} \mathrm{H}$ concentrations in ground water with decay-adjusted atmospheric concentrations.

\section{Dissolved Gases}

Water temperatures at the time of recharge can be calculated from $\mathrm{N}_{2} /$ Ar ratios (Heaton, 1981; Heaton and Vogel, 1981; Stute and Schlosser, 2000). The temperatures calculated for water from 10 wells (Table 1) range from about 5 to $10{ }^{\circ} \mathrm{C}$ (fig. 2). Concentrations of $\mathrm{N}_{2}$ and Ar that are larger than those of solubility equilibria alone are due to excess air (Stute and Schlosser, 2000). Excess air in volumes of as much as about $5 \mathrm{~cm}^{3}$ that occurred in certain samples and could have had two sources: (1) air bubbles that became entrained in ground water through natural fluctuations of the water table; or (2) air that became trapped during the sampling procedure. Regardless of the source of excess air, the temperatures indicated in figure 2 are considered reliable and were used in the calculation of CFC concentrations. The 30-year average air temperature in Oswego County is $8.7{ }^{\circ} \mathrm{C}$; therefore, 9 ${ }^{\circ} \mathrm{C}$ was assigned to samples in which $\mathrm{N}_{2} /$ Ar ratios were not measured. Elevations from the GPS unit were used to correct the temperatures calculated from $\mathrm{N}_{2} / \mathrm{Ar}$ ratios for atmospheric pressure.

\section{Isotopes}

Concentrations of ${ }^{3} \mathrm{H}$ in the 28 ground-water samples ranged from 0.8 to $30.5 \mathrm{TU}$ (Table 1). Of the 28 samples, 21 contained 21.3 to $30.5 \mathrm{TU}$, with an average of $25.6 \pm 2.4 \mathrm{TU}$. Qualitatively, these values indicate the presence of "some bomb ${ }^{3} \mathrm{H}$ " (Clark and Fritz, 1997, p. 185). The minimum concentration considered to represent comparatively unequivocal bomb ${ }^{3} \mathrm{H}$, that is post-1955 water, is $15 \mathrm{TU}$. Values of $\delta^{18} \mathrm{O}$ and $\delta \mathrm{D}$ in ground water (fig. 3) plot close to the local meteoric water line (LMWL). The deuterium excess value $d$ (defined as $\delta \mathrm{D}-8 \delta^{18} 0$, Dansgaard, $1964)$ averages 14.2. The large $d$ value for local meteoric water in relation to a value of 10 for the global meteoric water line may be due to snow that formed under non equilibrium conditions (Rozanski 


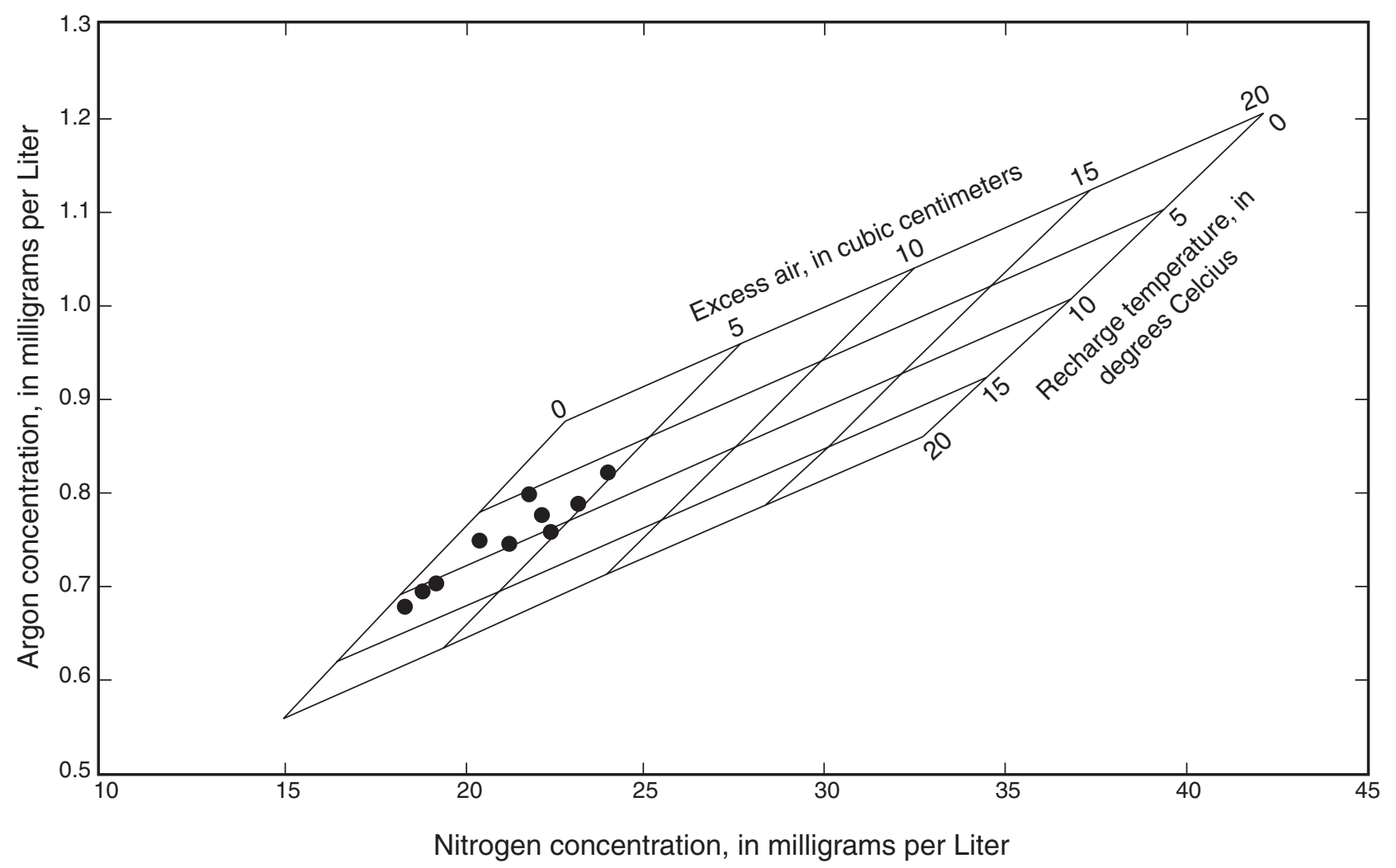

Figure 2. Argon concentrations and $\mathrm{N}_{2}$ concentrations in 10 ground-water samples from community wells in Oswego County, N.Y., and calculated water temperatures at time of recharge.

and others, 1993). The $\delta^{18}$ and $\delta \mathrm{D}$ values are consistent with local sources of recharge.

\section{Chlorofluorocarbons}

Three chlorofluorocarbon compounds, CFC-11 $\left(\mathrm{CFCl}_{3}\right), \mathrm{CFC}-12\left(\mathrm{CF}_{2} \mathrm{Cl}_{2}\right)$ and $\mathrm{CFC}-113\left(\mathrm{C}_{2} \mathrm{~F}_{3} \mathrm{Cl}_{3}\right)$ can be measured to determine ages of ground water. However, concentrations of CFC-12 were not used in this study because they exceeded modern concentrations in many samples; possible sources of excess CFC-12 include leaking septic systems, sewers, and underground storage tanks (Plummer and Busenberg, 2000). Three of the 28 samples (Camp Zerbe, Century House, and Hastings Inn; table 1) were omitted because they contained excessive CFC-11 concentrations, and the Lake Effect Inn sample could not be used because it contained degraded CFC-113. Trapped air can affect CFC ages in post-1990 samples (Plummer and Busenburg, 2000), but the samples contained comparatively small amounts, and the post1990 CFC-based ages are consistent with contemporaneous ${ }^{3} \mathrm{H}$ concentrations. This evidence suggests that post-1990 CFC-based ages are reliable.
Hydrodynamic dispersion usually is not a significant cause of incorrect CFC-based ages (Plummer and Busenburg, 2000), but it can affect ${ }^{3} \mathrm{H}$ concentrations (Plummer and others, 1993).

Ground-water recharge dates and ages (table 1) were estimated from plots of $\mathrm{CFC}-113$ versus $\mathrm{CFC}-11$ concentrations in air (fig. 4), and from plots of CFC-11 and $\mathrm{CFC}-113$ (fig. 5) versus ${ }^{3} \mathrm{H}$ concentrations in water. The plot of $\mathrm{CFC}-11$ versus ${ }^{3} \mathrm{H}$ is omitted because it is similar to the CFC- $113 /{ }^{3} \mathrm{H}$ plot. Samples that contained excess or degraded CFC-11 or CFC-113 are not represented on these plots. The recharge dates shown in each plot were obtained by projecting the compositions to the closest part of the atmosphericCFC line. Samples that plot within the shaded zone above the atmospheric-CFC line in figure 4 may represent mixtures of old and modern water. (The term "modern" water here means recharge dates of 1996 to 1999, whereas "old" is a relative term whose meaning varies with context.) Many of the ground-water compositions depicted in figure 5 plot above or below the atmospheric-CFC-113/ ${ }^{3} \mathrm{H}$ line. Some reasons for this may be (1) that the atmospheric ${ }^{3} \mathrm{H}$ values, which are mostly from Ottawa, Canada (the closest station), are different than those for Oswego County; (2) the 


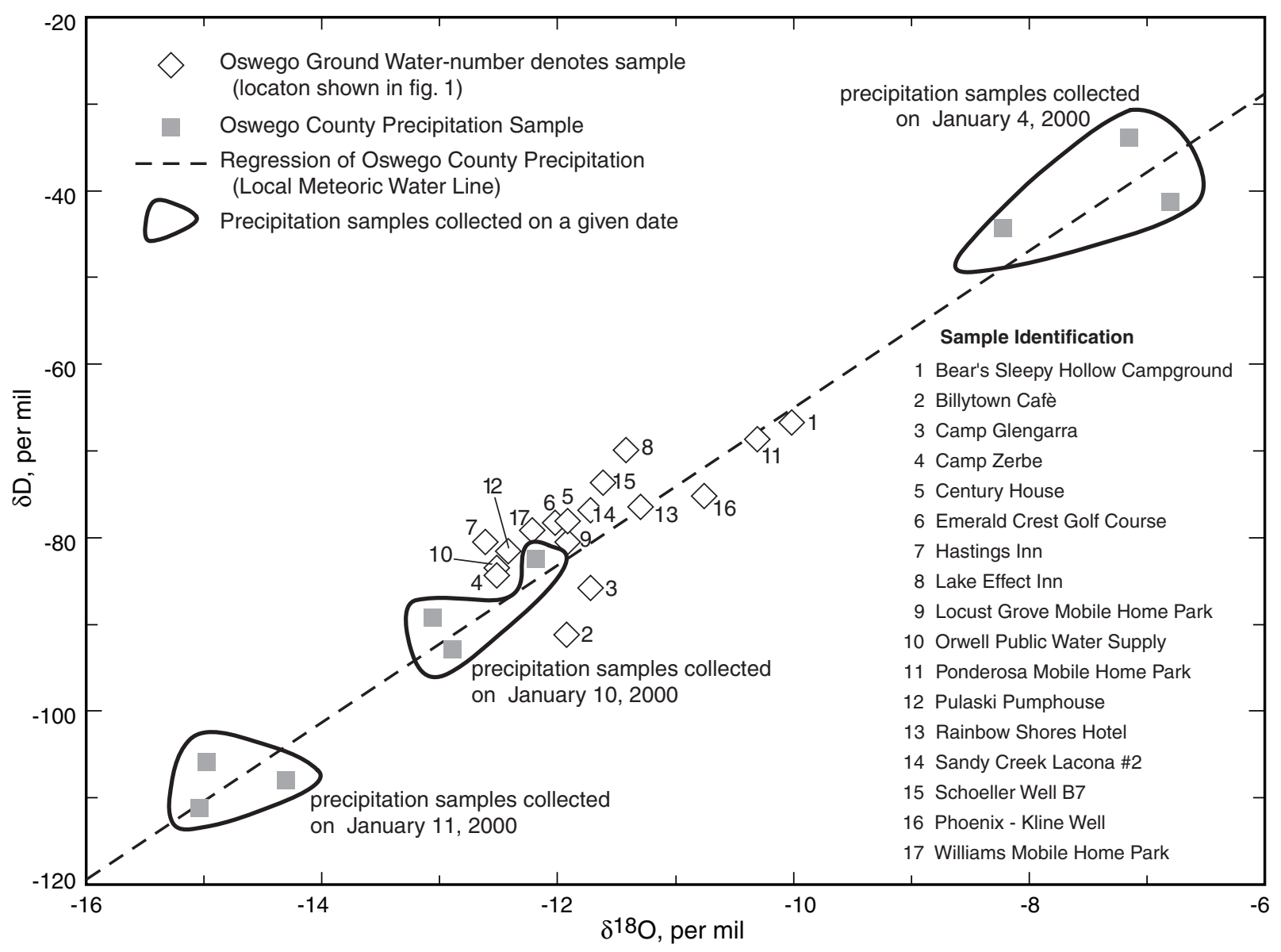

Figure 3. Oxygen- and hydrogen-isotope compositions of ground water and precipitation samples collected from selected sites in Oswego County, N.Y. (Well locations are shown in fig. 1.)

smoothing and averaging algorithm applied to the ${ }^{3} \mathrm{H}$ data was inappropriate (although simpler approaches produced curves similar to ones shown); and (3) points that lie off the CFC line represent mixtures of old and young water.

The range of recharge dates obtained for the ground water samples represented in figures 4 and 5 is variable, for example: Bear's Sleepy Hollow, $1957 \pm$ 1; Green Acres Mobile Home Park (MHP), 1957-67; Locust Grove MHP, 1987-92; Village of Mexico \#2, 1968-77; NIMO, 1967-76; Orwell Public water supply, 1988-93; Pulaski Pump house, 1973-80; and Sandy Creek Lacona \#2, $1981 \pm 2$. The recharge ages listed in table 1 are the differences between the midpoints of these ranges and 1999, the year of sample collection. CFC-based ages of ground water at the Billytown Cafe (fig. 1, table 1) varied with pumping time-from 44 years in the first sample to 14 years in the fifth sample, which was collected about 1 hour later. This pattern suggests that increasingly young (shallow) ground water was drawn into the well between collection of the first and fifth sample. Samples from Spruce Grove MHP on the Oneida Lake shore, and the Williams MHP just south of Lake Ontario (fig. 1), contained about 20 and 21 $\mathrm{mg} / \mathrm{L}$ of methane, respectively (table 1 ). Because CFCs are degraded in methanogenic environments (Plummer and Busenberg, 2000), the estimated ages of water from these two wells were based solely on ${ }^{3} \mathrm{H}$ concentrations.

\section{Relations Among Well Depth, Ground- water Ages, and Aquifer Lithology}

Most of the youngest water ( 8 to 14 years old) in this study came from wells shallower than $6.1 \mathrm{~m}$ that are completed in sand and gravel. Some sand-andgravel wells, however, that are $13.4 \mathrm{~m}$ deep or less contained water as old as 29 years (fig. 6, well 14), 
and one sand-and-gravel well $27.4 \mathrm{~m}$ deep contained water that was more than 40 years old (fig. 6,well 19). The oldest water was 40 to 44 years old and generally was found in samples from the fractured bedrock; the wells that produce from this unit are of moderate depth. The two highest specific conductance values obtained from aquifers of known lithology were in bedrock-well samples- $1,212 \mu \mathrm{S} / \mathrm{cm}$ at Ponderosa MHP and $954 \mu \mathrm{S} / \mathrm{cm}$ at Williams MHP. High specific conductance values would be expected to indicate water that has long been in contact with bedrock. Water from sand-and-gravel aquifers that directly overlie bedrock generally is of an age intermediate between that of old water from bedrock and young water from shallow parts of the sand-and-gravel aquifers. An exception is the Palermo Trailer Park well (fig. 6, well 21), which is the shallowest well completed in till, clay, silt, or fine sand but yields water that is 44 years old. The old age of this water is attributed to the low permeability and hydraulic conductivity of the fine-grained sediments and till near this well, which cause slow ground-water movement and long residence times within the unit. Water from three other wells finished in this type of aquifer (fig. 6 , wells 3,8 , and 16) may indicate a trend of increasing age with well depth.

\section{Source and Occurrence of Old Water}

The most significant result of this study is that sand-and-gravel aquifers can contain water 30 or 40 years old. Previously, sand-and-gravel aquifers in Oswego County were assumed to be recharged entirely by recent precipitation, seepage from streams, and other sources of young water, and numerical modeling of these aquifers typically used hydraulic boundaries that excluded contributions of old water from the fractured bedrock, till, and other aquifers

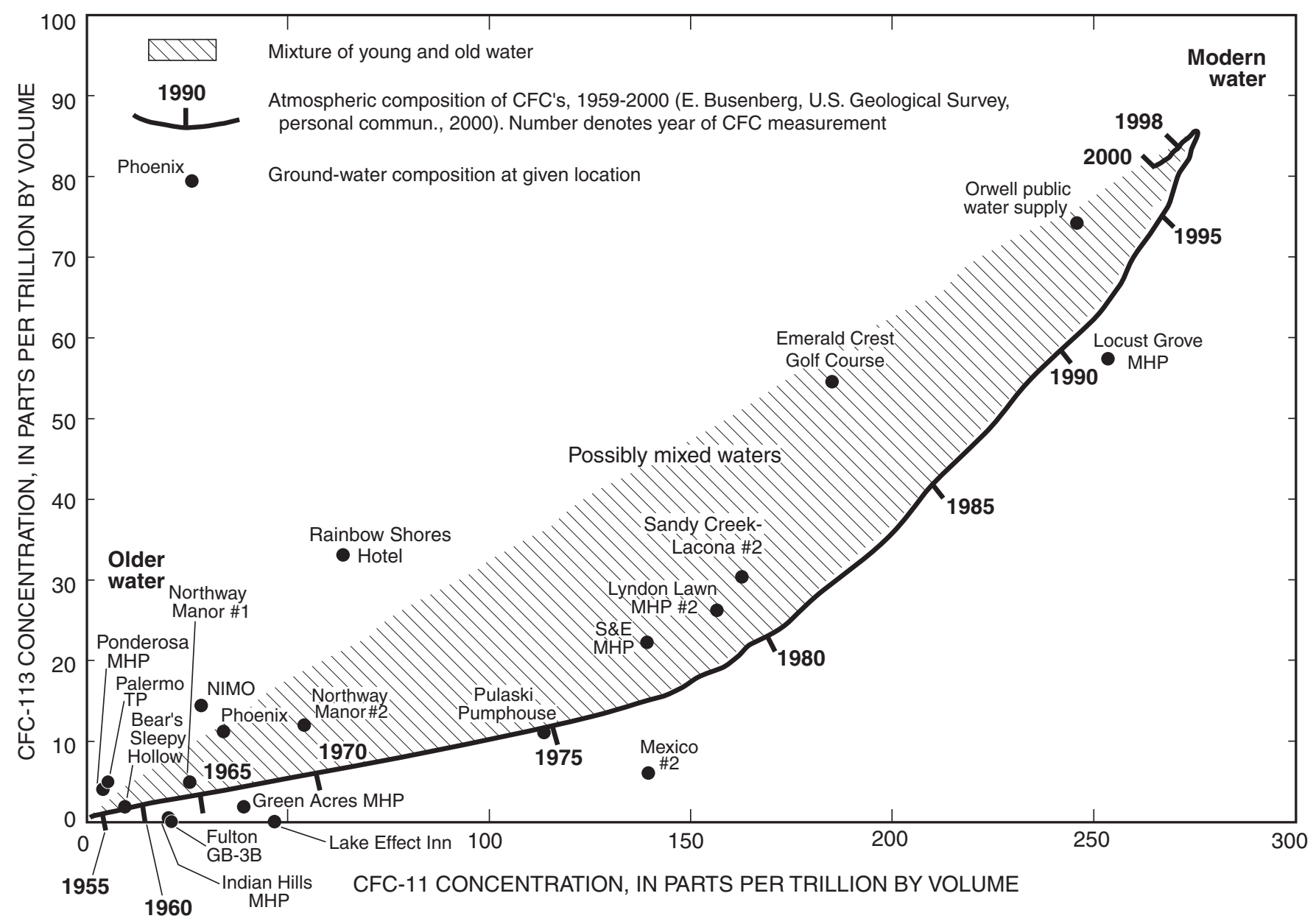

Figure 4. Comparison of CFC-11 and CFC-113 concentrations for ground water in Oswego County. Well locations are shown in fig. 1. 


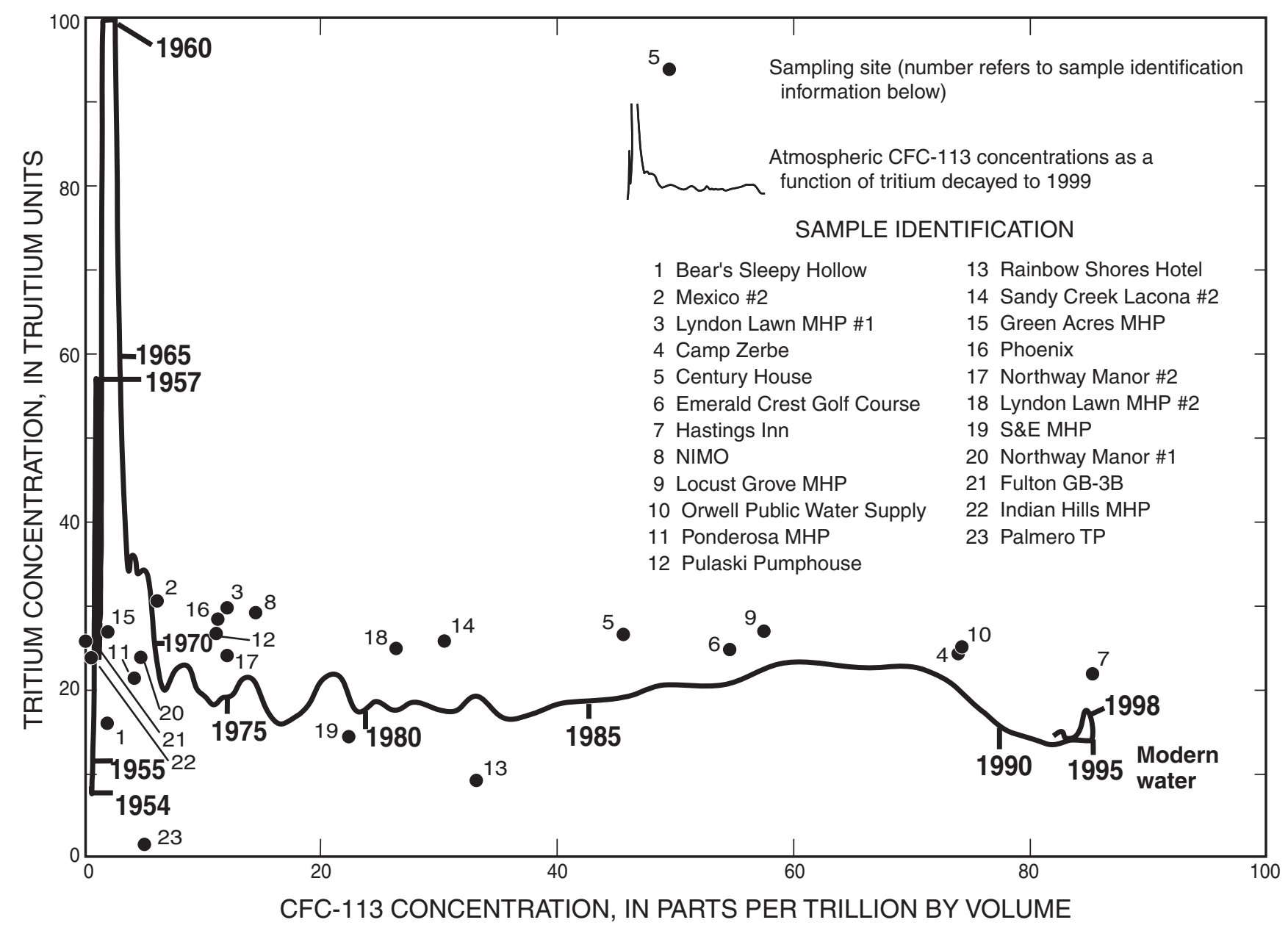

Figure 5. Comparison of CFC-113 and tritium concentrations. Atmospheric tritium concentrations are decayed to 1999. The curve is labeled with the dates corresponding to the atmospheric concentrations of CF-113 and tritium.

with low-hydraulic-conductivity. Sand-and-gravel aquifers that were simulated by numerical models to define ground-water-flow paths and traveltimes include those tapped by the Sandy Creek Lacona well, (Zariello, 1993); the Village of Phoenix - Kline well (Malcolm Pirnie Scott, internal document, 2000); and the Pulaski Pump House (Malcolm Pirnie Scott, internal document, 2000). These studies reported ground-water traveltimes equivalent to a recharge age of 3 to 5 years. These results may be correct for most local, shallow-flow systems but do not account for the ages of 30 to 40 years indicated by chemical dating of water from some wells.

Most old water probably has traveled through fractured bedrock, till, or clay-rich units. This older water may mix with younger water from sand-andgravel aquifers. Leakage from bedrock into sand-and- gravel aquifers can occur where the two units are hydraulically connected and is most likely where hydraulic heads in the bedrock aquifer are higher than in the overlying sediments, and the bedrock is highly fractured. Mixing might also occur where prolonged pumping draws down shallow, young water, as at the Billytown Cafe, or where pumping decreases the hydraulic head in surficial aquifers to levels below the potentiometric level in the underlying bedrock, allowing upward leakage from bedrock. Recharge of bedrock aquifers probably occurs mainly on the Tug Hill Plateau (fig. 1) and elsewhere in Oswego County where snowmelt and rain enter bedrock where it outcrops or is covered only by thin layers of permeable glacial sediments.

Mixing is a complex process that can homogenize water of a variety of ages from several aquifers and 


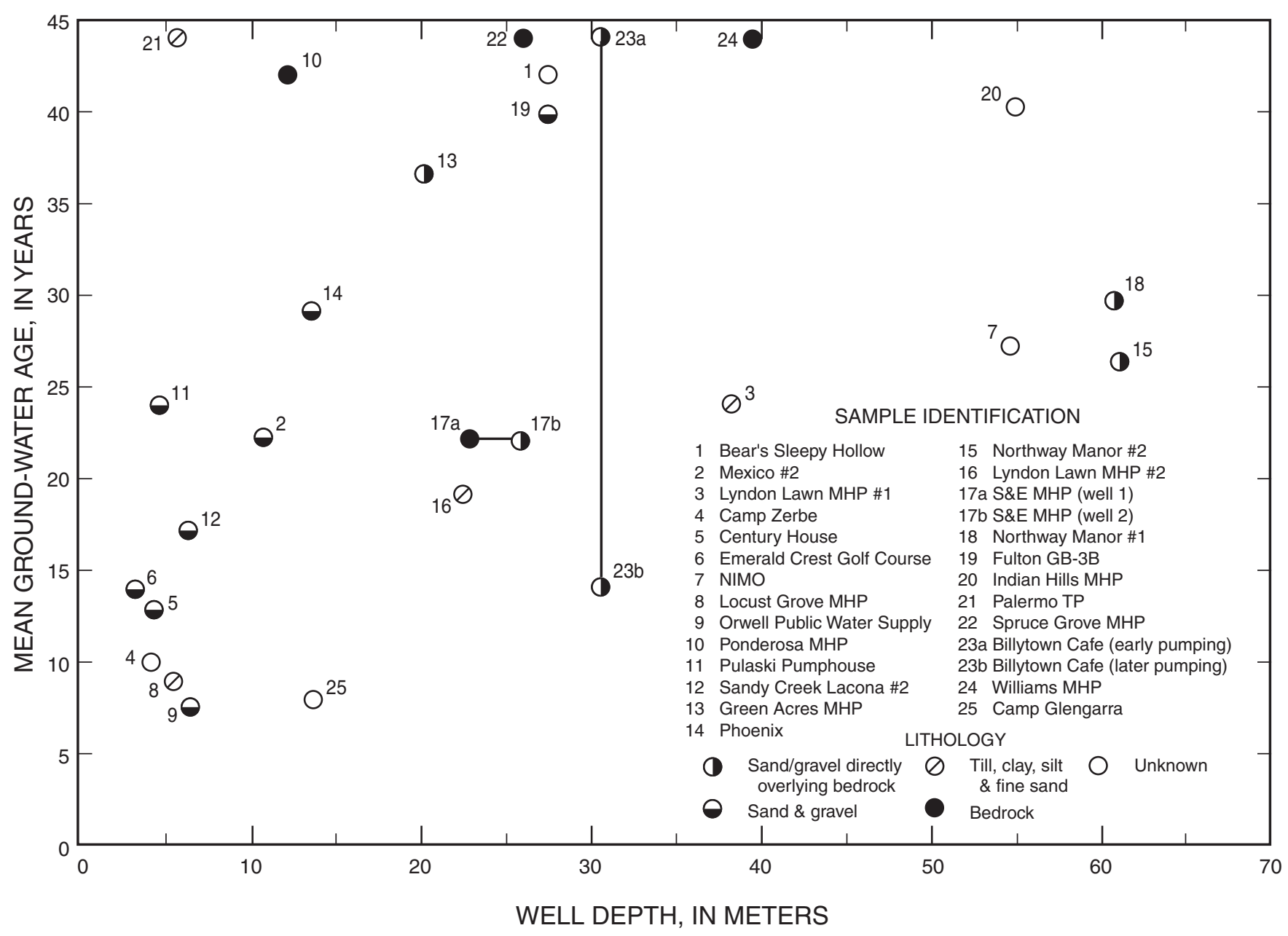

Figure 6. Mean ground-water age as a function of well depth and aquifer lithology, at the 24 wells sampled in Oswego County, N.Y., 1999. (Well locations are shown in fig. 1)

flow paths. Recharge dates in this study were not determined along well-defined flow paths in a single, homogenous aquifer. Therefore, the results cannot be used to rigorously identify the ground-water age or relative proportions of water from differing aquifers that may contribute to multicomponent mixtures. Samples that contain mixtures plot in the shaded area of figure 4. Binary mixing lines can be constructed for many samples in the shaded area, but their

correspondence to actual mixing processes probably is oversimplified. The well pair at S\&E MHP (fig. 4) provides a good example of mixed water. One well is completed in fractured bedrock, and the other is completed in overlying sand and gravel. The sample is a mix of water from the two wells and plots in the mixing zone in fig. 4 , which is consistent with the source of the sample.

A conceptual diagram of flow paths along an eastwest transect across Oswego County illustrates concepts presented in this report: young water associated with local flow paths, largely isolated from deeper, old water, recharges the shallow parts of some unconfined sand-and-gravel aquifers (A in fig. 7).

Parts of the same aquifers can contain old water whose flow has followed an intermediate path or possibly intersected fractured bedrock (B in fig. 7). Young and older water can mix in these aquifers where hydraulic conditions are favorable, as described earlier.

Comparatively young water also may recharge some fine-grained aquifers that consist of silty sand, silt, and clay that are tapped by shallow wells ( $\mathrm{C}$ in fig. 7).

Mixtures of young and old water can be obtained from wells that are completed in differing aquifers ( $D$ in fig. 7), such as the S\&E MHP well. Water of intermediate age can occur along local and subregional flow paths through clay, sand, and till aquifers (E in fig. 7). Finally, old water from bedrock aquifers can be obtained from some wells, particularly those near 


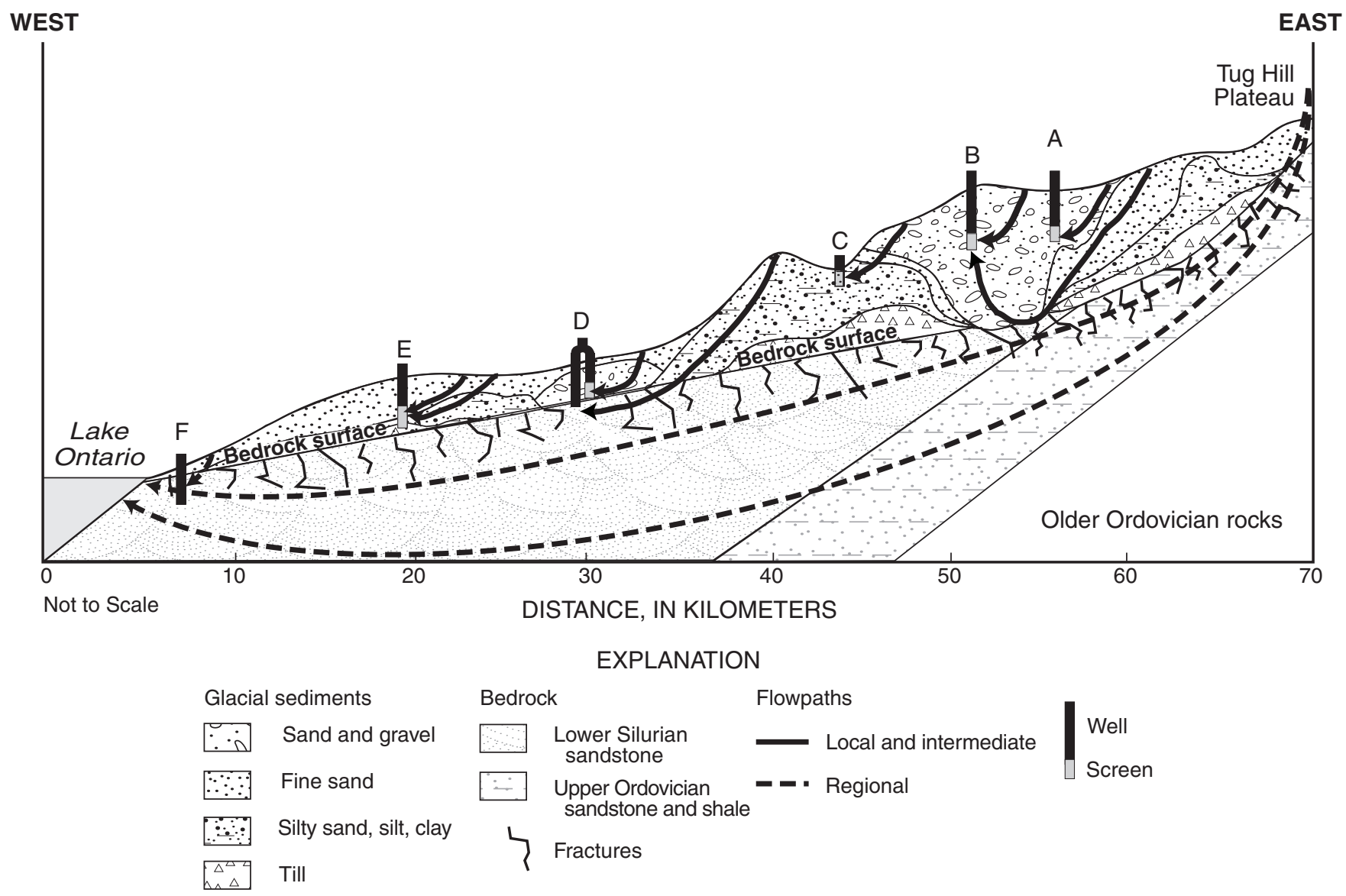

Figure 7. Conceptual east-west cross-section through Oswego County, N.Y., showing local and subregional flow paths, and various recharge scenarios.

regional discharge areas such as Lake Ontario ( $\mathrm{F}$ in fig. 7).

\section{Conclusions}

Many sand-and-gravel aquifers, the major water sources for many communities in Oswego County, contain mixtures of old and young water. Some aquifers in Oswego County contain old water that probably comes from regional flow paths that intersect fractured bedrock at the base of the glacial deposits and from poorly permeable deposits that retard ground-water movement. The quality of mixed water may be superior to that of young water because old ground water is less likely to contain contaminants in high concentrations.

Wellhead-protection efforts in Oswego County have concentrated on the unconfined sand-and-gravel aquifers that supply water to most communities, on the assumption that these aquifers are the most vulnerable to surficial and near-surface contaminants because aquifer recharge consists mostly of young water from direct precipitation and other surface sources. For example, excess CFC-12 concentrations were found in many wells in the study area, and the excess CFC-12 may be from surface or new-surface sources such as septic systems. Shallow wells or poorly constructed wells are particularly vulnerable to contamination.

The chemical age dating done in this study indicates that some unconfined sand-and-gravel aquifers contain mixed water from multiple sources with different recharge ages. The older water probably comes from regional flow systems in the underlying, fractured bedrock that discharge upward into the base of the glacial deposits and (or) from poorly permeable unconsolidated deposits that retard ground-water movement. Older water is less likely to contain high concentrations of human-derived contaminants because contaminants, if present, have more time to be reduced by natural processes, such as dilution, adsorption, filtration, and chemical degradation. 


\section{REFERENCES CITED}

Busenberg, Eurybiades, and Plummer, L.N.,1992, Use of chlorofluoromethanes $(\mathrm{CCl} 3 \mathrm{~F}$ and $\mathrm{CClF} 2)$ as hydrologic tracers and age-dating tools-the alluvium and terrace system of Central Oklahoma: Water Resources Research, v. 28, p. 2257-2283.

Clark, Ian, and Fritz, Peter, 1997, Environmental Isotopes in Hydrology: Boca Raton, Lewis Publishers, 529 p.

Dansgaard, W., 1964, Stable isotopes in precipitation, Tellus, v. 16, p. 436-468.

Dunkle, S.A., Plummer, L.N., Busenberg, Eurybiades, and others, 1993 Chlorofluorocarbons $\left(\mathrm{CCl}_{3} \mathrm{~F}\right.$ and $\mathrm{ClCl}_{2} \mathrm{~F}_{2}$ ) as dating tools and hydrologic tracers in shallow groundwater of the Delmarva Peninsula, Atlantic Coastal Plain, United States: Water Resources Research, v. 29, p. 3837-3860.

Harvey, F.E., D.L. Rudolph and S.K. Frape, 2000, Estimating ground water flux into large lakes: application in the Hamilton Harbor, western Lake Ontario, Ground Water, v. 38, 550-565.

Heaton, T.H.E., 1981, Dissolved gases: some applications to groundwater research, Transactions of the Geological Society of South Africa, v. 84, 91-97.

Heaton, T.H.E., and J.C. Vogel, 1981,'Excess' air in groundwater, Journal of Hydrology, v. 50, 201-216.

International Atomic Energy Association/World Meteorological Association, 1999, Global Network for Isotopes in Precipitation, The GNIP Database, Release 3, October 1999, URL: http://www.iaea.org/programs/ ri/gnip/gnipmain.htm.

Isachsen, Y.W., E. Landing, J.M. Lauber, L.V., Rickard, and W.B. Rogers, 2000, Geology of New York, New York State Museum Educational Leaflet, 28, 2nd edition, 294 p. and 3 plates.

Kantrowitz, I.H., 1970, Ground-water resources in the eastern Oswego River basin, New York, New York State Department of Environmental Conservation, Basin Planning Rept., ORB-2, 129 p.

Kaufman, S., and W.F. Libby, 1954, The natural distribution of tritium, Physical Review, v. 93, p. 1337-1344.

McDonald, M.G., and A.W. Harbaugh, 1988, A modular three-dimensional finite difference ground-water flow model, U.S. Geological Survey Techniques of WaterResources Investigations, Book 6, Chapter A1, 589 p.

Miller, T.S., 1982, Geology and-water resources of Oswego County, New York, U.S. Geological Survey WaterResources Investigations 81-60, $37 \mathrm{p}$.

Miller, T.S., D.A. Sherwood, and M.M. Krebs, 1989, Hydrogeology and water quality of the Tug Hill glacial aquifer in northern New York, U.S. Geological Survey Water-Resources Investigations 88-4014, 60 p.

Miller, T.S. and D.A. Sherwood, 1993, Geohydrology and water quality of the Sand Ridge glacial-drift aquifer in Oswego County, New York, U.S. Geological Survey Water-Resources Investigations 91-4042, 31 p.
Plummer, L.N., R.L. Michel, E.M. Thurman, and P.D. Glynn, 1993, Environmental tracers for age dating young ground water, in Regional Ground-Water Quality, edited by W.M. Alley, chap. 11, 255-294, Van Nostrand Reinhold, New York.

Plummer, L.N., and Busenberg, Eurybiades, 2000, Chlorofluorocarbons, in Environmental Tracers in Subsurface Hydrology, edited by P.G. Cook and A.L. Herczeg, chap. 15, 441-478, Kluwer, Boston.

Pollock, D.W., 1989, Documentation of computer programs to compute and display path lines using results from the U.S. Geological Survey modular three-dimensional finite-difference ground-water flow model, U.S. Geological Survey Open-File Report 89-381, 188 p.

Reilly, T.E., L.N. Plummer, Phillips, P.J., and Busenberg, Eurybiades, 1994, The use of simulation and multiple environmental tracers to quantify groundwater flow in a shallow aquifer, Water Resources Research, 30, 421-433.

Rozanski, K., L. Araguas, and R. Gonfiantini, 1993, Isotopic patterns in modern global precipitation, in Climate Change in Continental Isotopic Records, edited by P.K. Swart, K.C. Lohman, J. McKenzie, and S. Savin, chap. 1, 1-36, American Geophysical Union, Washington, D.C.

Soloman, D.K., and P.G. Cook, 2000, 3H and 3He, in Environmental Tracers in Subsurface Hydrology, edited by P.G. Cook and A.L. Herczeg, chap. 14, 397424, Kluwer, Boston.

Stute, M. and P. Schlosser, 2000, Atmospheric noble gases, in Environmental Tracers in Subsurface Hydrology, edited by P.G. Cook and A.L. Herczeg, chap. 11, 349377, Kluwer, Boston.

Szabo, Z., D.E. Rice, L.N. Plummer, Busenberg, Eurybiades, Drenkard, S. and Schlosser, P., 1996, Age dating of ground water using chlorofluorocarbons, tritium/helium3, and flow-path analysis, southern New Jersey coastal plain, Water Resources Research, 32, 1023-1038.

U.S. Environmental Protection Agency, 1974, Safe

Drinking Water Act; Public law 93-523 , 1986, Safe Drinking Water Act; Public law 93-339, section 1428.

Zariello, P.J., 1993, Determination of the contributing area to six municipal ground-water supplies in the Tug Hill glacial aquifer of northern New York, with emphasis on the Lacona-Sandy Creek well field, U.S. Geological Survey Water-Resources Investigation Report 90-4145, $51 \mathrm{p}$. 